\title{
Actiniaria des Südwestatlantik
}

\section{Sagartiidae und Metridiidae*}

\author{
K. RIEMANN-ZÜRNECK ${ }^{\text {*** }}$ \\ Institut für Meeresforschung; Bremerbaven, \\ und \\ Biologische Anstalt Helgoland; \\ Hamburg 50, Bundesrepublik Deutschland
}

\begin{abstract}
Actiniaria of the southwestern Atlantic Ocean. II. Sagartiidae and Metridiidae. Three species of Sagartiidae and a subspecies of Metridiidae are described from the shelf off Argentina. New anatomical data on Choriactis laevis (CARLGREN, 1899) are presented and peculiarities of the reproductive biology reported: (a) The species is hermaphroditic with complementary males - a situation not known from other seamanemones. (b) C. laevis is brood-caring; developmental stages remain in the gastric cavity of the parent up to the planula stage. (c) Planula organization corresponds to the "Edwardsia-stage" with a unique feature: an attachment organ develops before tentacles are formed; it is located at the aboral pole and contains masses of spirocysts. The organ derives from ectodermal material only, Carcinactis dolosa n. sp. greatly resembles representatives of the genus Calliactis (Hormathiidae). Carcinactis UchIDA, 1960 is compared with Verrillactis EngLand, 1971. For Phellia exlex (MCMURRich, 1904) additional descriptions of organization and cnidom are given. Metridium senile subsp. lobatum (CARLGREN, 1899) nov. comb. nov. grad., syn. Sagartia lobata CARLGREN, 1899, is the first record of a subspecies of Metridium senile in the southern hemisphere. For comparison, two specimens of Metridium senile from the North Atlantic have been investigated. Metridium exile HAND, 1955 is considered to represent a synonym of Metridium senile subsp. fimbriatum (VERRILL, 1865).
\end{abstract}

\section{EINLEITUNG}

Bei der Bestandsaufnahme der Bodenfauna des Südwestatlantik wurden von dem fischereitechnischen Forschungsschiff "WALTHER HERWIG" in den Jahren 1966, 1968 und 1970/71 insgesamt 708 Aktinien an 223 Stationen auf Schelf und Kontinentalabhang vor der Küste Argentiniens und Uruguays in Tiefen zwischen 30 und $1220 \mathrm{~m}$ gesammelt. In einer ersten Bearbeitung, welche auch in Methodik und Untersuchungs$\mathrm{XXXX}$

* Ergebnisse der Forschungsreisen des FFS „Walther Herwig" nach Südamerika Nr.

*t: Mitglied der Taxonomischen Arbeitsgruppe an der Biologischen Anstalt Helgoland. 
gebiet dieses Aktinien-Materials einführt, wurden sechs Arten der Familie Hormathiidae beschrieben (RIEMANN-ZürnECK, 1973). Die vorliegende Arbeit behandelt drei Arten der Familie Sagartiidae und eine Unterart der Familie Metridiidae.

Die besonderen taxonomischen Probleme der Sagartiidae und Metridiidae sollen in einem kurzen geschichtlichen Rückblick dargestellt werden: Zunächst waren die Typus-Gattungen beider Familien in der von Gosse (1858) geschaffenen Familie Sagartiidae (lapsus Sagartiadae) vereinigt. Später wurden die Sagartiidae durch das Hinzukommen zahlreicher verschiedenartiger Gattungen zu einem Sammelbegriff, der ein Synonym des modernen Taxon Acontiaria darstellte. Eine erste Gliederung der Familie nahm HADDON (1889) vor, der die dickwandigen, kutikulatragenden Formen (später ein Teil der Hormathiidae) abtrennte. Srmon (1892) spaltete jene Arten ab, die keinen mesodermalen Sphinkter besitzen (Arten vom Aiptasia-Typus), und Carlgren (1893) erkannte die unterschiedliche Anordnung der Gonaden und trennte eine Unterfamilie Metridiinae ab, welche neben Metridium noch die bei den Sagartiiden verbliebenen zartwandigen Gattungen der späteren Hormathiiden enthielt. Mit diesem Schritt war die für die Systematik der Acontiaria wichtige 'Trennung zwischen Sagartiiden und Hormathiiden vollzogen, wenngleich die Familie Hormathiidae im heutigen Sinne von CARLgren (1925, p. 6) geschaffen wurde. Nach der Abtrennung der Arten, welche Mikro- und Makrosepten besitzen und deren Nesselkapselausstattung HormathiidenMerkmale aufweist (Bathypelliidae CARLgREN, 1932, Isophelliidae StEPHEnson, 1935), blieben innerhalb der Sagartiidae acht Gattungen zurück.

Die Metridinae im Sinne Carlgrens (1893) erfuhren inzwischen eine Anhebung zur Familie Metridiidae (STEPHENSON, 1920), die verbunden war mit einer Erweiterung der Gruppe durch Gattungen vom Aiptasia-Typus. Die von CARLgRen (1932, p. 262) vorgeschlagene Abtrennung der Gattung Metridium als eigenständige Familie wurde schließlich von StepHenson (1935) vollzogen. Heute enthält die Familie neben der Typus-Gattung noch die Gattung Isometridium (CARLGREN, 1951), welche allerdings schon vom Erstbeschreiber als Genus incertae sedis angesehen wird.

\section{FAMILIE SAGARTIIDAE}

Nach Reduzierung des Gattungsbestandes der Sagartiidae auf acht Gattungen, welche weitgehend schon von Gosse (1858) berücksichtigt worden waren, blieb eine taxonomische Gruppe als unsicherer Rest mit vielen Schwierigkeiten für den Bearbeiter zurück. Der Status zahlreicher Arten ist auf Grund der vorliegenden Beschreibungen fraglich; da dies auch für einige Typus-Arten gilt, sind auch einige Gattungen in Frage gestellt. Es muß nun mit dem Versuch begonnen werden, die inzwischen auf 14 Gattungen angewachsene Familie einer Revision zu unterziehen. Hierbei sollte neben der umfassenderen Untersuchung der Tiere eine kritische Uberprüfung der taxonomischen Wertigkeit der Merkmale angeschlossen werden. Die nachfolgenden Beschreibungen sind der Versuch, dieser Forderung gerecht zu werden; bei der Untersuchung der Tiere wird besonderes Gewicht gelegt auf Merkmale der Verdauungsorgane und der Gonaden. 


\section{Gattung Choriactis}

Der Typus der Gattung, Choriactis impatiens (DANA, 1849), muß nach den vorliegenden Beschreibungen (Dana, 1849, p. 135; McMurrich, 1904, p. 272; Carlgren, 1927, p. 77) als fragwürdige Art angesehen werden. Es ist darüber hinaus nicht auszuschließen, daß die Flachwasser-Art C. impatiens mit der meist aus größeren Tiefen stammenden $C$. laevis identisch ist. Da eine genaue Beschreibung des Typus-Fundortes existiert (DANA, 1849, p. 136), ist eine spätere Überprüfung des Sachverhaltes möglich.

\section{Choriactis laevis (CARLGREN, 1899)}

Carlgren (1927, p. 79, Fig. 51, 52); syn. Sagartia laevis Carlgren (1899, p. 35); syn. Choriactis crassa McMurrich (1904, p. 274, Pl. 17, Fig. 62-64, Pl. 18, Fig. 65), Stephenson (1920, p. 548); syn. Choriactis crassoides Pax (1922, p. 90), Pax (1923, p. 23, P1. 2, Fig. 5); syn. Choriactis opalescens $\operatorname{PAx}$ (1922, p. 91, Pl. 1, Fig. 5). Synonymisierungen von CARLGREN (1927, p. 79).

M a t e ria l. 124 Tiere von 68 Stationen der" "WALther-Herwig“-Reisen 1966 und 1970/71. Anatomische und histologische Untersuchungen wurden an netn Tieren durchgeführt (Tab. 1),

Habitu s. (a) Körperform und Größe (Abb. 1a-c): Choriactis laevis ist eine relativ große Sagartide; ihr Habitus ist in fixiertem Zustand stumpf kegelförmig, seltener zylindrisch. Fußscheibe stets breiter als der Körperdurchmesser, an festen Gegen-

\section{Tabelle 1}

Anatomisch untersuchte Individuen von Choriactis laevis

\begin{tabular}{|c|c|c|c|c|c|}
\hline $\begin{array}{l}\text { Tier } \\
\text { Nr. }\end{array}$ & $\begin{array}{l}\text { Walther- } \\
\text { Herwig- } \\
\text { Expedition }\end{array}$ & $\begin{array}{c}\text { Station } \\
\mathrm{Nr} .\end{array}$ & Koordinaten & $\begin{array}{l}\text { Tiefe } \\
\text { (m) }\end{array}$ & $\begin{array}{l}\mathrm{Abb} . \\
\mathrm{Nr} .\end{array}$ \\
\hline $65 / 66$ & 1966 & 303 & $45^{\circ} 13^{\prime} \mathrm{S} 60^{\circ} \mathrm{O} 2^{\prime} \mathrm{W}$ & 310 & \\
\hline $98 / 66$ & 1966 & 302 & $44^{\circ} 07^{\prime} \mathrm{S} 60^{\circ} 05^{\prime} \mathrm{W}$ & 220 & $1 \mathrm{c}$ \\
\hline $122 / 66$ & 1966 & $390\}$ & $38^{\circ} 56^{\prime} \mathrm{S} 55^{\circ} 31^{\prime} \mathrm{W}$ & 300 & \\
\hline $\begin{array}{l}119 / 66 \\
116 A\end{array}$ & $\begin{array}{l}1966 \\
1970 / 71\end{array}$ & $\begin{array}{l}390 \\
116\end{array}$ & $37^{\circ} 57^{\prime} \mathrm{S} 55^{\circ} 19^{\prime} \mathrm{W}$ & 150 & \\
\hline $\begin{array}{l}110 \pi \\
128\end{array}$ & $1970 / 71$ & 128 & $40^{\circ} 00^{\prime} \mathrm{S} 58^{\circ} 03^{\prime} \mathrm{W}$ & 100 & $2 a$ \\
\hline 186 & $1970 / 71$ & 186 & $46^{\circ} 00^{\prime} \mathrm{S} 60^{\circ} 55^{\prime} \mathrm{W}$ & 123 & $2 b$ \\
\hline 213 & $1970 / 71$ & 213 & $50^{\circ} 00^{\prime} \mathrm{S} 62^{\circ} 54^{\prime} \mathrm{W}$ & 152 & \\
\hline 327 & $1970 / 71$ & 327 & $42^{\circ} 58^{\prime} \mathrm{S} 58^{\circ} 58^{\prime} \mathrm{W}$ & 800 & \\
\hline
\end{tabular}

ständen angeheftet, die immer biogenen Ursprungs sind (Mollusken- und Brachiopodenschalen, Korallen-Skelette und bewohnte Röhren sedentärer Polychaeten). Tentakelkranz unterschiedlich stark kontrahiert, bei der Mehrzahl der Tiere Tentakel äußerlich sichtbar.

(b) Mauerblatt glatt, bei großen Tieren mit schwachen Vertiefungen. Marginaler Abschnitt verdickt, durch eine ringförmige Einsenkung vom übrigen Mauerblatt leicht abgesetzt, bei vollständig kontrahiertem Tentakelkranz mit meist 12 unregelmäßigen 

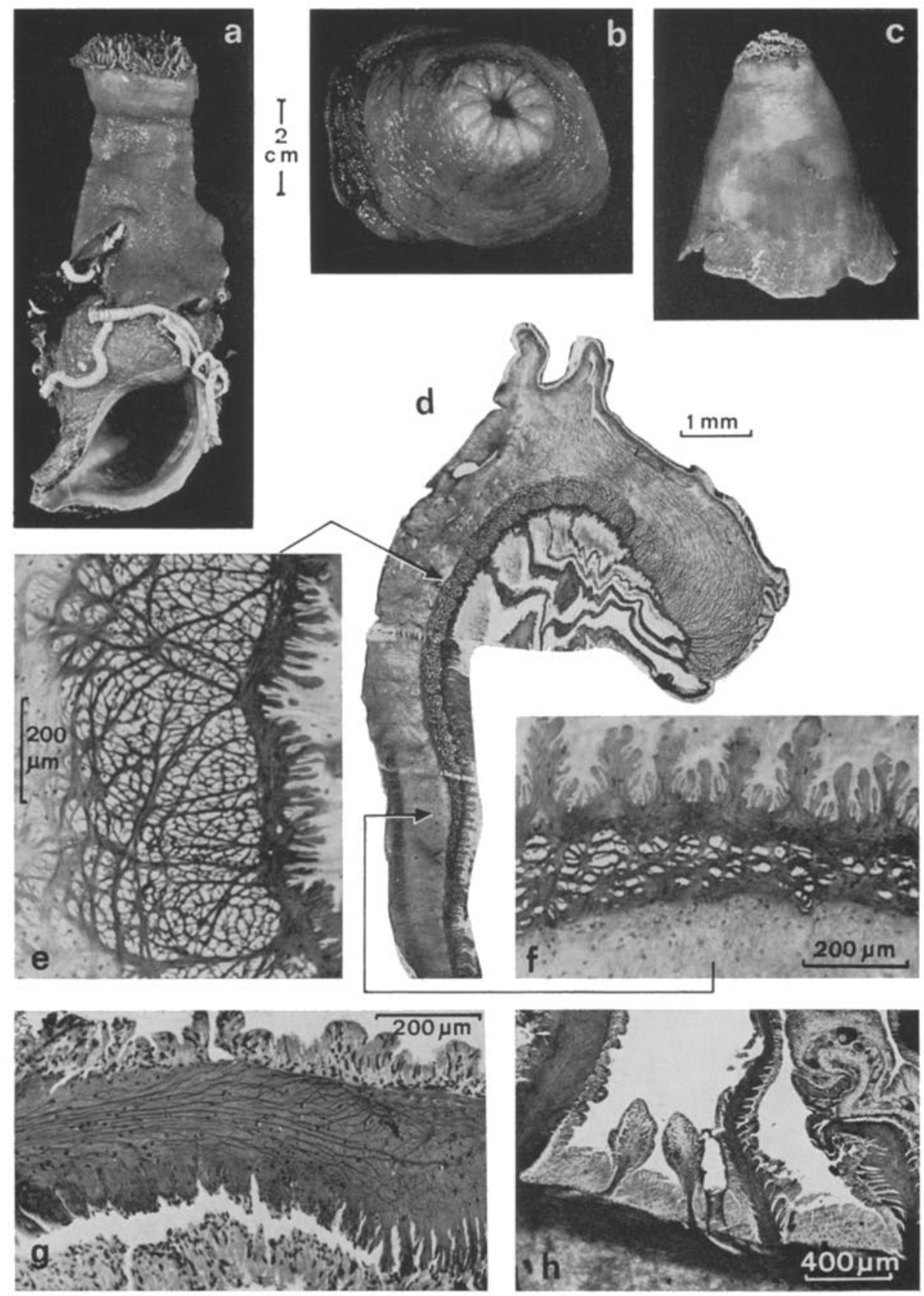

Abb. 1: Choriactis laevis. a Tier 139; $b$ Tier 299; c Tier 98/66; $d$ Mesogloealer Sphinkter; $e$ und $f$ Vergrößerte Ausschnitte des mesogloealen Sphinkters; $g$ Substruktur der Septenmesogloea (1. Cyklus); $h$ Parietalmuskulatur des 4. und 5. Septencyklus 
Marginalwülsten. Cincliden nicht vorhanden. Ektoderm leicht abfallend, darunter (insbesondere in Limbusnähe) sind die Insertionen der Septen sichtbar. Fußscheibenrand gelappt, jedoch niemals ungeschlechtliche Vermehrung durch Lazeration.

(c) Gewicht der größten Tiere ca. $50 \mathrm{~g}$, Gewicht der kleinsten Tiere ca. $10 \mathrm{~g}$.

(d) Färbung: Frisch fixierte Tiere lassen das Vorhandensein mehrerer Farbvarietäten erkennen. Nach McMurrich (1904, p. 275) gibt es orange, gelbe, rosa und weiße Tiere. Bei den fixierten Tieren des vorliegenden Materials ist der Margin immer heller als der übrige Körper.

$\mathrm{B}$ a u p 1 a n. Körperwand für eine Sagartiide relativ dick, 1 bis $2 \mathrm{~mm}$, im marginalen Abschnitt bis $4 \mathrm{~mm}$.

Tentakel in mehr als 6 Cyklen vorhanden (mehr als 192 Tentakel); sie sind dünn und die innersten sind bis $1,5 \mathrm{~cm}$ lang; Fangtentakel nicht vorhanden.

Septen in 5 Cyklen regelmäßig hexamer angeordnet, füntter Cyklus nicht komplett vorhanden. 12 oder 24 Septenpaare (Cyklus 1 und 2 oder Cyklus 1 bis 3) vollständig. Gonaden an allen Septen der Cyklen 1 bis 4 mit Ausnahme der beiden Direktivenpaare. 5. Septencyklus ohne Anhangsorgane, bei einem großen Tier mit trilobaten Filamenten. Alle Septen mit dicker Mesogloea. Die Mitte des Gastralraumbodens wird von 6 Septenpaaren strahlenförmig erreicht.

Actinopharynx mit 2 gutentwickelten Siphonoglyphen und 24 oder 48 regelmäßigen Längswülsten, die auf Querschnitten eine Höhe von 1 bis $1,5 \mathrm{~mm}$ haben und sich über den Insertionen der vollständigen Septen befinden (Abb. 2a, b). Acontien lang und relativ dick, mit einem Durchmesser von $200 \mu \mathrm{m}$.

$\mathrm{H}$ is to $\log$ i e. (a) Cnidom: Bei sechs Tieren wurde die Nesselkapselausstattung untersucht; bei 19 Tieren wurde die Variabilität von Typen und Abmessungen der Nesselkapseln bestimmt. Die Variabilität der Nematocysten ist bei Choriactis laevis gering, eine größere Variationsbreite zeigen lediglich die Abmessungen der AcontienNematocysten: p-Mastigophoren: 52-87 $\mu \mathrm{m}$, Basitrichen 1: 36-55,5 $\mu \mathrm{m}$, Basitrichen 2: 14,5-26 $\mu \mathrm{m}$. Eine Abhängigkeit von der Körpergröße oder von den Fundorten der Tiere existiert nicht. In Tabelle 2 werden die Nesselkapseln des Tieres Nr. 213 angegeben (vgl. Abb. 3); die Typen und Abmessungen der Nesselkapseln dieses Tieres entsprechen jenen, die bei der Mehrzahl der untersuchten Tiere gefunden wurden. Die mit * bezeichneten Nesselkapseln waren bisher bei dieser Art nicht bekannt (vgl. CARLGREN 1927, pp. 80, 82). Besonderheiten des Cnidoms: (1) p-Mastigophoren des Mauerblattes nur im marginalen Abschnitt; (2) Unilobate Mesenterialfilamente mit drei verschiedenen p-Mastigophoren.

Abb. 2: Choriactis laevis. a Querschnitt durch das Tier Nr. 128 im Bereich des Actinopharynx. Die römischen Ziffern bezeichnen die Septenpaare des 2. bis 4. Cyklus; $b$ Querschnitt des Actinopharynx eines kleineren Tieres (186); $c$ Innerster Abschnitt eines gonadentragenden Septums. Die Zeichnung zeigt den Querschnitt eines trilobaten Filamentes und die Einstillpung des Ovarialepithels uber dem Nucleus der Oocyte; $d$ Karte der Fundorte von Choriactis laevis, wobei eng benachbarte Fundorte schwarz gekennzeichnet sind; $e, f, g$ Habitus, Querschnitt und Längsschnitt der Planula von Choriactis laevis. DO - Dotter, CIL.T. - Ciliärer Trakt, CNID. T. - Cnidularer Trakt, EK - Ektoderm, EN - Entoderm, FIL - Mesenterialflamente, GON - Gonaden, HA - Haftorgan der Larve, MES - Mesogloea, MU - Mundöf. nung, NUC - Nucleus mit Nucleolus, PH - Actinopharynx, RET.T. - Retikulärer Trakt, TA Tentakelanlage, VS - Ventrolaterales Septum, WI - Wimperepithel 

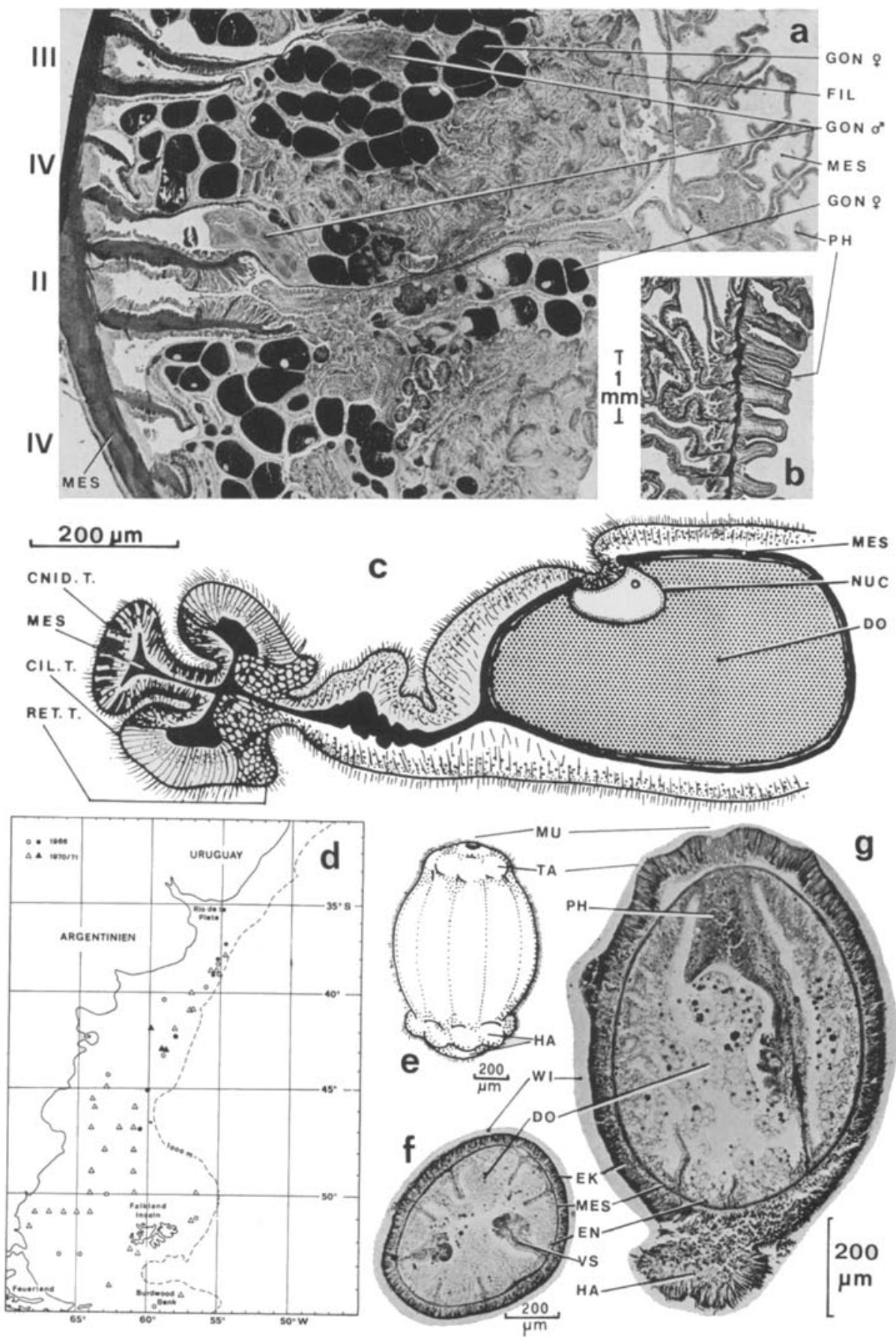
Tabelle 2

Abmessungen der Nesselkapseln von Choriactis laevis (Tier Nr. 213)

\begin{tabular}{|lll|}
\hline Körperregion & Nematocysten & Dimension $(\mu \mathrm{m})$ \\
\hline Mauerblatt & p-Mastigophoren* & $28-32,5 \times 4,5-5$ \\
& Basitrichen 1 & $21-27 \times 2,5$ \\
& Basitrichen $2^{*}$ & $13-17,5 \times 2-2,5$ \\
Tentakel & Spirocysten maximal & $41,5 \times 5$ \\
& p-Mastigophoren & $32,5-41,5 \times 4-5$ \\
& Basitrichen 1 & $23,5-28,5 \times 2-2,5$ \\
& Basitrichen 2* & $15,5-18 \times 2$ \\
Actinopharyox & p-Mastigophoren 1 & $43-51 \times 5,5-6$ \\
& p-Mastigophoren 2 & $27-29 \times 4-5$ \\
& Basitrichen 1 & $35-39 \times 2,5$ \\
& Basitrichen 2 & $17-19 \times 2$ \\
Mesenterialfilamente & p-Mastigophoren 1* & $47-52 \times 5,5-6$ \\
(unilobat) & p-Mastigophoren 2* & $27 \times 3-4$ \\
& p-Mastigophoren $3^{*}$ & $12-14,5 \times 4,5$ \\
& Basitrichen* & $17-19,5 \times 2$ \\
Acontien & p-Mastigophoren & $73-80,5 \times 5,5-6,5$ \\
& Basitrichen 1 & $47-54,5 \times 2,5-3$ \\
& Basitrichen 2*5 & $17-22 \times 2$ \\
\hline
\end{tabular}

(b) Muskulatur: Längsmuskulatur der Tentakel ektodermal, Radialmuskulatur der Mundscheibe ektodermal, Mesogloea-Fortsätze verzweigt. Retraktoren der vollständigen Septen gleich stark entwickelt, die des 3. Cyklus proximal schwächer werdend und schließlich wie an den Septen des 4. Cyklus nur noch als unbedeutende Mesogloea-Fortsätze zu erkennen; Septen des 5. Cyklus ohne Retraktoren. Direktiven mit besonders krä̈tigen Retraktoren. Parietalmuskulatur an den Septencyklen 1 bis 3 nicht und an den Cyklen 4 und 5 schwach vorhanden (Abb. 1h). Ringmuskulatur der Körperwand ungewöhnlich stark entwickelt, Mesogloea-Fortsätze fingerförmig und auf Längsschnitten 60 bis $150 \mu \mathrm{m}$ hoch, auch im untersten Abschnitt des Sphinkters vorhanden, von diesem durch einen Mesogloeastreifen getrennt (Abb. 1d, f). Marginaler Sphinkter (Abb. 1d) mesogloeal, kräftig, 0,7 bis 1,3 cm lang, größte Breite ca. $1 \mathrm{~mm}$. Der Sphinkter ist auf seiner ganzen Länge vom Entoderm durch einen Streifen verdichteter Mesogloea getrennt. Im obersten, breitesten Abschnitt nimmt der Sphinkter die ganze Breite der Mesogloea ein.

(c) Mesogloea der Körperwand und der größeren Septen mit gitter- bis netzförmiger Substruktur (Abb. 1g). Rundliche Einschlüsse in der Mesogloea häufig, besonders in den Actinopharynx-Wülsten.

(d) Gonaden bei allen Tieren vorhanden, bei den im Jahre 1966 - vom 21. Juli bis 3. August - gesammelten Tieren in reifem Zustand. Reife Eier haben einen Durchmesser von 500 bis $600 \mu \mathrm{m}$; sie sind dotterreich und bleiben auch nach langer Lagerung in Alkohol orange bis rotbraun gefärbt. Die Versorgung der Oocyten mit Nährstoffen erfolgt über eine Einstïlpung des Ovarial-Epithels über dem Kern der Oocyte (Abb. 2c). Hier dringt das Epithel bis unter die das Ei umschließende Mesogloea vor und verursacht eine Einwölbung der Eioberfläche. Die Sexualverhältnisse bei Choriactis laevis 


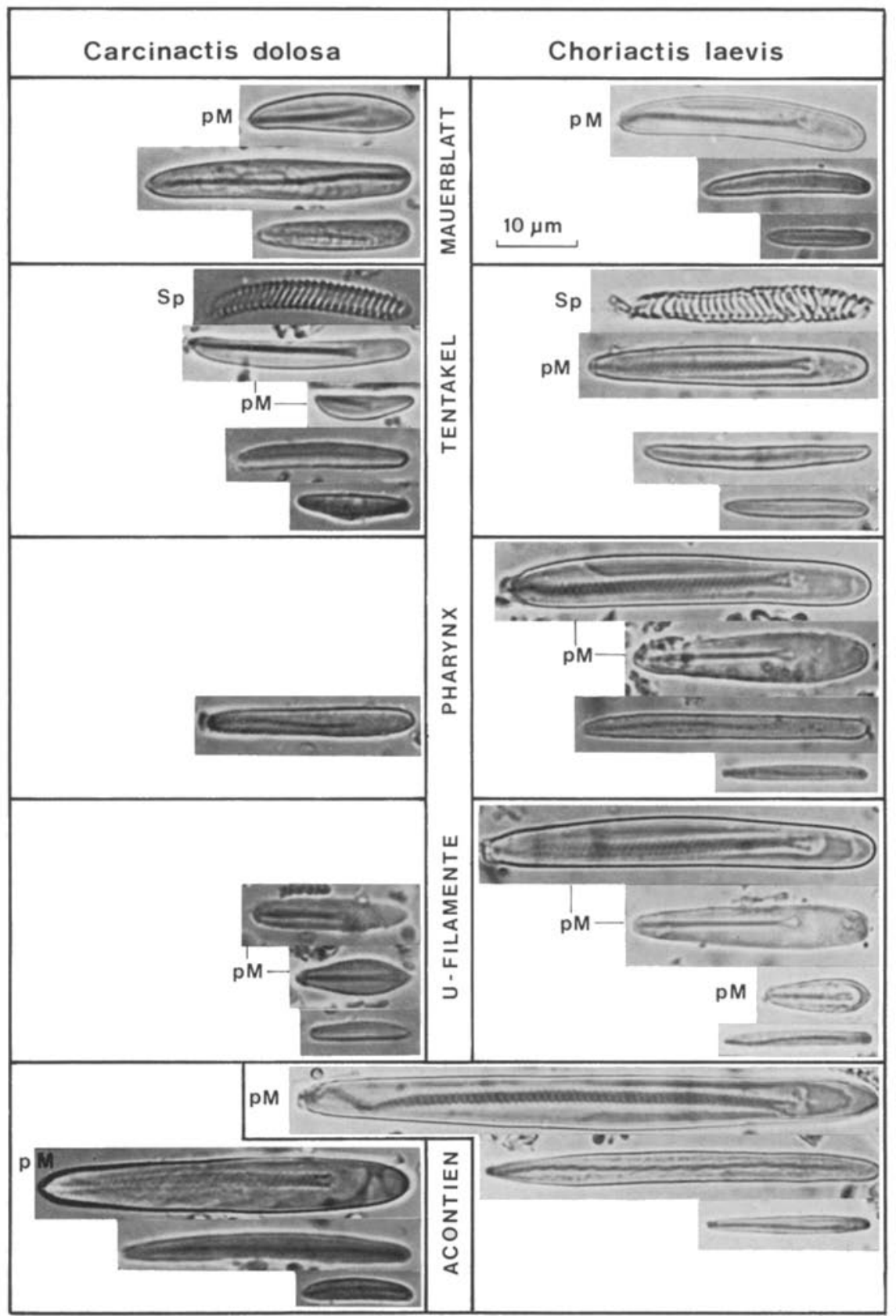

Abb. 3: Nesselkapselausstattung von Carcinactis dolosa n. sp. (Tier Nr. 68/60) und Choriactis laevis (Tier Nr. 213). Sp - Spirocyste, $\mathrm{pM}$ - p-Mastigophore; alle anderen abgebildeten Nesselkapseln sind Basitriche 
sind ungewöhnlich und bisher innerhalb der Actiniaria unbekannt: Die Art ist zwittrig mit komplementären Männchen. Von 55 untersuchten Tieren erwiesen sich nur 5 als Männchen; alle anderen Tiere wurden zunächst nach makroskopischer Betrachtung als Weibchen eingestuft. Eine genaue Untersuchung zeigte jedoch, daß alle diese Tiere an einigen wenigen Septen Hodenfollikel tragen und somit $Z$ witter sind (Abb. 2a).

(e) Trilobate Filamente: Form und histologischer Aufbau sind in Abbildung 2c dargestellt.

Verbreitung. Choriactis laevis kommt auf dem gesamten argentinischen Schelf vor und kann somit als eine der charakteristischen Tierarten dieses Gebietes angesehen werden. Ihre nördliche Verbreitungsgrenze hat die Art am Südrand des „Riode-la-Plata “-Deltas, im Süden reicht ihre Verbreitung über die von „WALTHER HER WIG festgestellten Fundorte (Abb. 2d) hinaus bis zu den Südshetland-Inseln (PAx, 1923, pp. 21, 23: beschrieben als Choriactis crassoides und Ch. opalescens). Die vertikalen Verbreitungsgrenzen der Art können an Hand der "WALTHER-HER WrG “-Stationen nicht sicher bestimmt werden, da nur wenige Stationen in den Randgebieten des Kontinentalabhanges liegen. Unter diesem Vorbehalt ergibt sich aus den wenigen Daten, daß C. laevis in Meerestiefen von weniger als $100 \mathrm{~m}$ nicht mehr und in Tiefen unter $500 \mathrm{~m}$ nur selten gefunden wird. Eine entsprechende Tiefenverbreitung der Art ergibt sich aus der Literatur; es gibt jedoch einen Hinweis, daß C. laevis im subantarktischen Teil ihres Verbreitungsgebietes auch in geringen Tiefen vorkommt (CARLgREN, 1899, p. 36).

Brutpflege und Embryonalentwicklung. Choriactis laevis ist eine brutpflegende Sagartiide; zehn von 33 im Jahre 1966 gesammelten Tiere tragen in ihren Gastralräumen (insbesondere in dem von Fußscheibe und Mauerblatt gebildeten äußersten Winkel des Gastralraumes) Hunderte von sich entwickelnden Eiern oder Embryonen. Bei acht Tieren sind die Eizellen ohne äußerlich sichtbare Veränderungen, in einem Tier (Nr. 201/66) finden sich neben solchen Eiern Furchungsstadien (Morulae) und in einem anderen Tier (Nr. 123/66) Larven, die dem Entwicklungsstadium der Planula entsprechen.

Die frühe Entwidklung von Choriactis laevis ergibt sich danach wie folgt: Befruchtung der Eier im Gastralraum, möglicherweise bereits in den Septen. Furchung der Eioberfläche erst nach der 6. oder 7. Kernteilung; es entsteht eine Morula, deren etwa 100 Blastomeren mit der zentralen Dottermasse kommunizieren. Typus der Entodermbildung unbekannt. Planula (Abb. 2e, f, g) mit 8 Primär-Septen (Edwardsia-Stadium), die beiden ventrolateralen Septen entstehen als erste. Die am weitesten entwickelten Larven sind 1,5 mm lang, sie sind kräftig bewimpert, und ihr Dottervorrat ist aufgezehrt. Sie besitzen die Anlagen folgender Organe: Actinopharynx, Mesenterialfilamente an den ventrolateralen Septen und eine noch mit Sekret verschlossene Mundöffnung. Am oralen Pol hat sich das Ektoderm in Form von acht kleinen Erhebungen von der Mesogloea gelöst: Dies sind die Anlagen der 8 Primär-Tentakel (Abb. 2e, g). Eine Besonderheit der Choriactis-Larve ist das auch bei jüngeren Larven vorhandene aborale Haftorgan. Wegen seines besonderen Aufbaues und der massenhaft vorhandenen Spirocysten* bezeichne ich dieses Organ nicht als Fußscheibe. Das Haftorgan ent-

* Die Spirocysten kommen meist nur in den Tentakeln der Aktinien vor; ihre Haftwirkung gegenüber Nahrungspartikeln, aber auch gegenüber bevorzugten Siedlungs-Substraten ist seit langem bekannt (vgl. STEPHENSON, 1928, p. 60). 
steht als ringförmiger Wulst, der sich auf Schnitten als eine Aufwölbung des Ektoderms darstellt (Abb. 2g). Später hebt sich auch das Ektoderm innerhalb des Ringwulstes von der Mesogloea $a b$, und es entsteht ein äußerlich der Fußscheibe ähnliches Organ, an dessen Bildung sich jedoch, wie gesagt, nur das Ektoderm beteiligt. Die enthaltenen Spirocysten haben dic maximalen Abmessungen $47 \times 3,5 \mu \mathrm{m}$. Das übrige Ektoderm der Planula ist mit p-Mastigophoren $(17,5-26 \times 3,5-4,5 \mu \mathrm{m})$ und in der Nähe der Mundöffnung mit Basitrichen $(38 \times 3 \mu \mathrm{m})$ ausgestattet, die Tentakelanlagen enthalten junge, noch nicht funktionsfähige Spirocysten. Neben den Spirocysten des Haftorgans und den jungen Spirocysten der Tentakel, die sich beide - wie für Spirocysten bekannt - gegenüber Farbstoffen basophil verhalten, finden sich einzelne, über den ganzen Larvenkörper verteilte Spirocysten, die sich histochemisch entgegengesetzt verhalten (acidophil).

Differentialdiag nose. Relativ große Sagartiide, die mit breiter Fußscheibe auf festen Gegenständen biogenen Ursprungs sitzt. Mauerblatt glatt, für eine Sagartiide dickwandig; marginaler Abschnitt verdickt, etwas heller gefärbt und bei kontrahiertem Tentakelkranz mit 12 unregelmäßigen Marginalwülsten. Keine Cincliden. Die Art kommt in mehreren Farbvarietäten vor. Septen mit dicker Mesogloea, die eine gitter- bis netzförmige Substruktur zeigt. Actinopharynx mit 24 oder 48 Längswülsten und mit ungewöhnlich zahlreichen Nesselkapseln ausgestattet. p-Mastigophoren des Mauerblattes nur im marginalen Abschnitt, dort aber massenhaft vorhanden; unilobate Mesenterialfilamente mit drei verschiedenen $\mathrm{p}$-Mastigophoren. Retraktoren und Parietalmuskulatur der Septen schwach ausgebildet, Ringmuskulatur der Körperwand und mesogloealer Sphinkter kräftig und von charakteristischem Aufbau. Die Art ist zwittrig mit komplementären Männchen, das zahlenmäßige Verhältnis von Männchen : Zwitter verhält sich wie $1: 10$. Reife Oocyten orange bis rotbraun gefärbt, dotterreich; ihre Versorgung mit Nährstoffen erfolgt über eine Einstülpung des Ovarialepithels. Fortpflanzungszeit im Südwinter (Juni bis August); die Art ist brutpflegend, wobei sich die Entwicklungsstadien insbesondere in dem von Mauerblatt und Fußscheibe gebildeten äußersten Winkel des Gastralraumes befinden. Choriactis laevis hat ein sehr großes Verbreitungsgebiet, das den gesamten argentinischen Kontinentalabhang, die Burdwood-Bank und einige antarktische Inseln einschließt. Die bevorzugten Meerestiefen liegen zwischen 100 und $500 \mathrm{~m}$.

$\mathrm{D}$ is k us s io n. Die drei Arten der Gattung Choriactis, C. impatiens (DANA 1849, Typus der Gattung), C. subantarctica PAx, 1922 und C. laevis (CARLgRen, 1899), gehörten bisher zu jenen Sagartiiden-Arten, deren Morphologie besonders wenig bekannt und deren taxonomische Zuordnung fraglich war. Obwohl dies auch heute noch für die Typus-Art C. impatiens gilt, erlauben doch die an Choriactis laevis gewonnenen Untersuchungsergebnisse eine Diskussion des Status der Gattung.

Carlgren (1949, pp. 101, 104) hat der Gattung Choriactis innerhalb der Sagartiiden eine isolierte Stellung zugewiesen, ohne hierfür eine ausreichende Begründung zu geben. Die Untersuchungsergebnisse von Choriactis laevis bestätigen, daß es sich hier um den Vertreter einer Gattung handelt, die gegenwärtig mit keiner anderen Sagartiiden-Gattung in Verbindung zu bringen ist und deren Sonderstellung daher gerechtfertigt erscheint. Entscheidendes Gewicht bei dieser Eingruppierung kommt meiner Ansicht nach dem nunmehr zweifelsfrei festgestellten Befund zu, daß bei Choriactis laevis 
keine Cincliden vorhanden sind. Innerhalb der Sagartiidae fehlen Cincliden sonst nur noch bei der Gattung Phellia, die im übrigen keine vergleichbaren Merkmale besitzt. Darüber hinaus können die ungewöhnlichen Sexualverhältnisse und die mit der Brutpflege in Zusammenhang stehenden morphologischen Besonderheiten der Larvalentwicklung von taxonomischer Bedeutung sein.

\section{Gattung Carcinactis}

\section{Carcinactis dolosa n. sp.}

Material. 25 Tiere von zwei Stationen der "WALTHER-HerwIG"-Expedition 1968. Mit Ausnahme zweier Tiere, von denen sich das eine (68/60) auf einem Stein und das andere (68/74) auf einem Schneckengehäuse angesiedelt hat, sitzen alle Tiere auf Muschelschalen. Holotypus: Tier Nr. 68/60 (Abb. 4b, e, f, k).

\section{Tabelle 3}

Material von Carcinactis dolosa n. sp. (WALTHER-HER wig-Expedition 1968)

\begin{tabular}{|lccc|}
\hline Tier Nr. & Station Nr. & Koordinaten & Tiefe $(\mathrm{m})$ \\
\hline $\begin{array}{l}68 / 57,68 / 59 \\
68 / 66,68 / 68 \\
68 / 73,68 / 75\end{array}$ & 55 & $31^{\circ} 06^{\prime} \mathrm{S} 50^{\circ} 36^{\prime} \mathrm{W}$ & 40 \\
\hline $68 / 74$ & 76 & $26^{\circ} 47^{\prime} \mathrm{S} 47^{\circ} 52^{\prime} \mathrm{W}$ & 40 \\
\hline
\end{tabular}

Ha b itus. (a) Körperform und Größe (Abb. 4a-c): Sagartiide von mittlerer Größe, die äußerlid Ahnlichkeit hat mit Arten der Gattung Calliactis (Familie Hormathiidae). Körperdurchmesser 2 bis $4 \mathrm{~cm}$, Körperhöhe 1 bis $4,5 \mathrm{~cm}$, Größenunterschiede zwischen den einzelnen Tieren gering. Tentakelkranz stets kontrahiert, Körperöffnung radiärsymmetrisch; bei zwei weniger stark kontrahierten Tieren sind an der Körperöffnung einige Tentakel sichtbar. Körperform je nach Kontraktionszustand zylindrisch, kegelförmig oder halbkugelig. Die Fußscheibe ist, wenn es die Größe der Unterlage zuläßt, gegenüber dem Mauerblatt verbreitert. Rand der Fußscheibe unregelmäßig ausgebuchtet, nicht auf dem Untergrund festgeheftet, mit durchscheinenden Septen-Insertionen.

(b) Mauerblatt: In zwei Regionen, Scapus und Scapulus, gegliedert. Scapus glatt, mit Ausnahme des Fußscheibenrandes relativ dickwandig, mit zahlreichen Querfurchen.

Abb. 4: Carcinactis dolosa n. sp. a Tiere Nr. 68/57; b Holotypus (Tier Nr. 68/60); $c$ Tier Nr. 68/74; $d$ Tentakelquerschnitt (Tier Nr. 68/57); $e$ Trilobates Mensenterialfilament des Holotypus (68/60); $f$ Mesogloealer Sphinkter des Holotypus (68/60); $g$ Septenpaar des 1. Cyklus und Radialmuskulatur der Mundscheibe (Tier Nr. 68/57); $h$ Marginaler Abschnitt des mesogloealen Sphinkters; $i$ Proximaler Abschnitt des mesogloealen Sphinkters und Ringmuskulatur der Körperwand; $k$ Parietalmuskulatur der Septen des Holotypus: links Septum des 4. Cyklus, Mitte Septenpaar des 5. Cyklus, rechts Septum des 3. Cyklus. BAS - Basitriche, EK - Ektoderm, EN - Entoderm, MES - Mesogloea, PIG - Pigmenteinschlüsse 

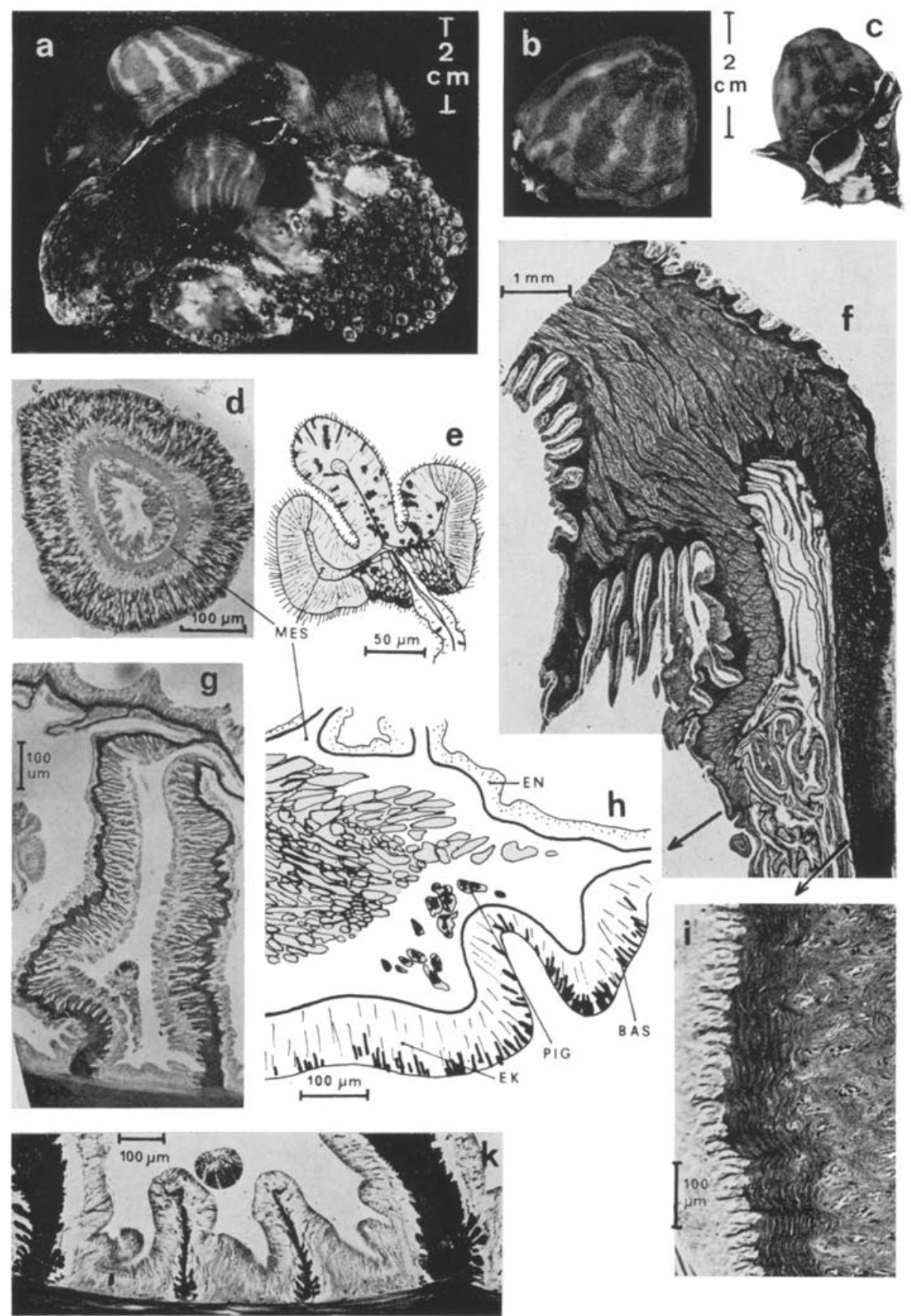
Scapus ohne Kutikula, höchstens im basalen Abschnitt mit leicht abfallenden Sandkörnern und kleinen Fremdpartikeln. Cincliden in einem eng begrenzten Abschnitt am Rande der Fußscheibe ( 1 bis $3 \mathrm{~mm}$ vom Limbus entfernt), über den Endocoelen der größeren Septenpaare, in einem Fall konnten 48 Cincliden gezählt werden. Die Cincliden sind klein und schwer zu erkennen, am besten dann, wenn ihre Umgebung etwas dunkler gefärbt ist oder Acontien austreten. Scapulus glatt und dünnwandiger als der Scapus, of durch eine schwache Vertiefung vom Scapus abgesetzt, mehr oder weniger stark in den Körper eingeschlagen und mit zahlreichen feinen Längsfurchen, die zahlenmäßig den Insertionen der Septen entsprechen, je nach Kontraktionszustand 48 oder 96 Längsfurchen.

(c) Farbmuster am lebenden Tier unbekannt; die vorliegenden fixierten Tiere zeigen jedoch nach etwa zweijähriger Lagerung in $75 \%$ Alkohol auffallende graubraune Farbmuster, die nur bei einigen Exemplaren fehlen (Abb. 4a-c); insgesamt erinnern die Farbmuster wiederum an die einer Calliactis. Der Scapus der Tiere trägt 12 breite Längsbänder, die zum Limbus hin spitz und zum Scapulus hin stumpf auslaufen; Breite der Längsbänder individuell variabel, bei einigen Tieren im mittleren Abschnitt des Mauerblattes miteinander verschmelzend (Abb. 4a). Am Limbus sind zwischen die 12 Längsbänder 12 Flecke eingefügt, die ebenfalls zum Limbus hin spitz auslaufen. Bei einigen Tieren sind am Rand zum Scapulus 12 feine Streifen zwischen die Bänder eingefïgt, in der Regel verschmelzen hier jedoch die Farbmuster zu einer einheitlichen Färbung. Scapulus mit Ausnahme des dunkel gefärbten Margin farblos.

B a u p l a n. (a) Tentakel sehr zahlreich, etwa 382, d. h. in 7 Cyklen vorhanden. Hexamere Anordnung der Tentakal undeutlich, da sie dichtgedrängt auf mehreren Loben (meist 6) der Mundscheibe angeordnet sind. Tentakel kurz und spitz, die innersten größer als die äußeren, bis $3 \mathrm{~mm}$ lang. Apex der Tentakel bei vielen Tieren dunkel pigmentiert (Pigment befindet sich im Entoderm), es kommen jedoch auch farblose und pigmentierte Tentakel innerhalb eines Tentakelkranzes vor. Die größten Tentakel können während der Kontraktion in sich selbst zurückgezogen werden. Durch die Kontraktion des umfangreichen Tentakelkranzes wird der mittlere Abschnitt der Mundscheibe tief in den Gastralraum gedrückt.

(b) Septen in 5 Cyklen regelmäßig hexamer angeordnet; am Limbus ist auch der 6. Septencyklus in Form winziger kurzer Septen vorhanden. Erster bis dritter Septencyklus vollständig, dritter Cyklus jedoch nicht mit der ganzen Länge des Actinopharynx verwachsen. Vollständige Septen mit ovalen Marginalstomata, die sich nahe am Mauerblatt in der Höhe des proximalen Sphinkter-Endes befinden. Mundscheibe in Verbindung mit 48 Septenpaaren, Entoderm der Mundscheibe dunkel pigmentiert. Actinopharynx farblos, dïnnwandig, unregelmäßig gefurcht, mit zwei bilateral angeordneten Siphonoglyphen und Direktivenpaaren. Anordnung der Gonaden: Unterhalb der Mundscheibe besitzt zuerst der 4. Cyklus Gonaden und trilobate Filamente, im mittleren Abschnitt des Actinopharynx auch der 3. Septencylklus. Nahe dem proximalen Ende des Pharynx ist der 2. Cyklus mit schwach entwickelten Gonaden und unilobaten Filamenten ausgestattet; der erste Cyklus ist ganz oder teilweise steril, Gonaden hier nur in Form einiger Follikel vorhanden, an den Direktiven fehlend. Bei einigen Tieren besitzt auch der kleinste Septencyklus (5.) im untersten Abschnitt des Mauerblattes Gonaden und trilobate Filamente, wobei oft nur ein Septum eines Paares 
Gonaden entwickelt (Tier 68/60). Acontien kurz, an allen Septen mit Ausnahme der kleinsten (5. und 6. Cyklus) vorhanden.

$\mathrm{H}$ i s t o log i e. (a) Cnidom (vgl. Abb. 3): Die Nesselkapselausstattung wurde bei den Tieren 68/55, 68/57 und 68/60 untersucht; bei sechs weiteren Tieren wurden die Nesselkapseln aus Tentakeln und Acontien untersucht und vermessen. Besonderheiten der Nesselkapselausstattung sind: (1) Breite Basitrichen im Mauerblatt, die besonders dicht gedrängt im Scapulus der Tiere vorkommen (an Schnittpräparaten zu erkennen, Abb. 4h). (2) Das Fehlen ron p-Mastigophoren im Actinopharynx. (3) Das Entoderm der Tiere enthält kleine tropfenförmige $\mathrm{p}$-Mastigophoren; diese Nesselkapsel fehlt in den Mesenterialfilamenten, im Pharynx und in den Acontien.

Tabelle 4

Abmessungen der Nesselkapseln von Carcinactis dolosan. sp.

\begin{tabular}{|lll|}
\hline Körperregion & Nematocysten & Dimension $(\mu \mathrm{m})$ \\
\hline Mauerblatt & p-Mastigophoren & $12-19 \times 4-4,7$ \\
einschließlich Scapulus & Basitrichen 1 & $27,5-36,5 \times 4-5$ \\
& Basitrichen 2 & $12-17 \times 3$ \\
Tentakel & Spirocysten maximal & $23,5-26 \times 4-4,5$ \\
& p-Mastigophoren 1 & $22-28,5 \times 2,5-4$ \\
& p-Mastigophoren 2 & $8-14,5 \times 3-4,5$ (Entoderm) \\
& Basitrichen & $18-23,5 \times 2-2,5$ \\
Actinopharynx & Basitrichen & $19,5-34 \times 2,5-3,5$ \\
Mesenterialfilamente & p-Mastigophoren 1 & $18-23,5 \times 3-4$ \\
(unilobat) & p-Mastigophoren 2 & $10-13,5 \times 4-5$ \\
& Basitrichen & $9-13,5 \times 2$ \\
Acontien & p-Mastigophoren & $39-54,5 \times 5-6$ \\
& Basitrichen 1 & $22-32 \times 2-2,5$ \\
& Basitrichen 2 & $12-17 \times 1,5-2$ (selten) \\
\hline
\end{tabular}

(b) Muskulatur: Längsmuskulatur der Tentakel ektodermal, Mesogloea-Fortsätze auf Querschnitten der Tentakel unregelmäßig geformt, höchstens so lang wie die Dicke der Tentakel-Mesogloea. Retraktoren an den Septencyklen 1 bis 4 vorhanden, diffus bandförmig (Abb. 4g), ohne „bäumchenartige" Abschnitte, an den Cyklen 1 bis 3 im Gonadenbereich schwach circumskript diffus. Querschnitte im Bereich des Actinopharynx zeigen, daß die Retraktoren an den Cyklen 1 bis 3 auf $2 / 3$ der Septenoberfläche vorhanden sind, wobei sie nicht in der Mitte der Septen, sondern nahe am Mauerblatt angeordnet sind, wo die Mesogloea-Lamelle etwas verdickt ist. Retraktoren der Direktivenpaare schwächer entwickelt, wenig verzweigt, Mesogloea jedoch dicker als bei anderen Septen des 1. Cyklus. Radialmuskulatur der Mundscheibe mesoektodermal (Abb. 4g), ein Retikulum bildend, das als gleichmäßige Schicht die nur halb so dicke Mundscheiben-Mesogloea überzieht. Parietalmuskulatur im obersten Abschnitt des Körpers an den Septen nicht vorhanden, im unteren Teil der Pharynx-Region schwach ausgebildet, bilateral symmetrisch in Form weniger fingerförmiger MesogloeaFortsätze vorhanden (Abb. 4k); die Parietalmuskulatur fehlt an den Endocoel-Seiten der Direktiven. Ringmuskulatur der Körperwand im Scapus krättig entwickelt 
(Abb. 4i), im Scapulus fehlend; Mesogloea-Fortsätze auf Längsschnitten bis $50 \mu \mathrm{m}$ lang, wenig verzweigt. Die Ringmuskelstränge scheinen an der Körperwand spiralig zu verlaufen, da sich auf Längs- wie auch auf Querschnitten durch die Körperwand ähnliche histologische Bilder ergeben. Marginaler Sphinkter (Abb. 4f) mesogloeal, in beiden Körperabschnitten vorhanden, je nach Kontraktionszustand des Scapulus 1 bis $1,8 \mathrm{~cm}$ lang. Struktur des Sphinkters wenig variabel, proximal nahe dem Entoderm mit sehr kleinen, weit auseinanderliegenden Alveolen beginnend, die sich zum Scapulus hin vergrößern und zu dicht beieinander liegenden Gruppen zusammenschließen, die beim Übergang zum Scapulus fast die gesamte Breite der Mesogloea ausfüllen und nur jeweils einen schmalen Streifen zum Ektoderm und zum Entoderm hin freilassen (Abb. 4f). Im Scapulus vergrößern sich die Alveolen weiter, während die MesogloeaBalken zwischen den Alveolen und Alveolen-Gruppen schmäler werden und schließlich distal ein Retikulum bilden.

(c) Mesogloea des Mauerblattes mit faserförmigen Einschlüssen, die sich auf der dem Gastralraum zugewandten Hälfte der Mesogloea verdichten. Die Mesogloea des Scapulus weist darüber hinaus in dem zum Ektoderm gewandten alveolenfreien Streifen im marginalen Bereich Pigment-Einschlüsse auf (Abb. $4 \mathrm{~h}$ ), die $\mathrm{zu}$ der bereits beschriebenen dunklen Färbung des Margin führen.

(d) Histologische Besonderheiten der Epithelien: Ektoderm des Scapus gelegentlich schwach eingesenkt, mit einer Anhäufung flaschenförmiger Drüsenzellen, deren Inhalt auch nach der Azanfärbung ungefärbt bleibt. Diese Mauerblatt-Differenzierungen sind selten und unterscheiden sich in ihrem histologischen Bild von den bei den Gattungen Sagartia und Cerews bekannten "Saugwarzen“, so daßs ich diesen Strukturen keine taxonomische Bedeutung bei der Zuordnung dieser Art beigemessen habe.

(e) Gonaden bei allen untersuchten Tieren vorhanden. Die Eizellen erreichen auf Schnitten einen Durchmesser von $100 \mu \mathrm{m}$. Die Art ist getrenntgeschlechtlich. Das vorliegende Tier-Material zeigt keine Hinweise auf ungeschlechtliche Vermehrung.

Verbre itung. C. dolosa wurde an zwei Stationen im Sublitoral des subtropischen Südwestatlantik gesammelt; die Fundorte liegen etwa $450 \mathrm{~km}$ voneinander entfernt und befinden sich beide in $40 \mathrm{~m}$ Tiefe. Das Fundort-Gebiet gehört hinsichtlich seiner Aktinien-Fauna zu den besonders schlecht untersuchten Meeresgebieten. Diese Region wurde von FFS "WALTHER HERwIG" nur während der vorzeitig abgebrochenen Reise im Jahre 1968 untersucht; da nur an sechs Stationen Aktinien gefunden wurden, konnten nur wenig neue Daten über die Arten und ihre Verbreitung in diesem Gebiet gesammelt werden. Uber die Verbreitung von C. dolosa kann zur Zeit festgestellt werden, daß ihr Vorkommen an das Vorhandensein von Mollusken-Schalen gebunden scheint. Die Art ist vergesellschaftet mit einer Calliactis spec.; die.beiden Arten zeigen habituelle Ubereinstimmungen. Eine vergleichbare Vergesellschaftung ist von den beiden äußerlich ebenfalls ähnlichen Arten Calliactis polypus (FOrSKaL) und Verrillactis paguri (VERRILL) bekannt, wobei diese Arten of gemeinsam ein von einem Einsiedlerkrebs bewohntes Schneckengehäuse besiedeln (vgl. ENGLAND, 1971).

Differentialdiagnose. Mittelgroße Sagartiide, die habituell und morphologisch Merkmale der Gattung Calliactis mit Sagartiiden-Merkmalen verbindet. Mauerblatt in Scapus und Scapulus gegliedert, Scapus für eine Sagartiide relativ dickwandig. Tentakel zahlreich (etwa 384, d. h. 7 Cyklen), kurz und spitz, die inneren nur 
wenig größer als die äußeren. Septen in 5 Cyklen, 1. bis 3. Cyklus vollständig. Gonaden am besten entwickelt an den Cyklen 3 und 4, am 2. Cyklus schwach, am 1. ganz oder teilweise fehlend. Mundscheibe undulat mit meist 6 Loben, Mundscheibe und Tentakel voll kontrahierbar. Scapus mit auffallendem Farbmuster. Scapulus mit Anhäufungen yon basitrichen Nesselkapseln und Pigment-Einschlüssen im marginalen $\mathrm{Ab}$ schnitt der Mesogloea (Abb. 4h). Verbreitung: Sublitoral des subtropischen Südwestatlantik; die Art bevorzugt als Substrat Mollusken-Schalen.

Diskussion. Die Einordnung der hier beschriebenen Sagartiide in die von ToHru Uchida (1960, pp. 595-596) aufgestellte Gattung Carcinactis erfolgte wegen der weitgehenden Ubereinstimmung mit dem Typus der Gattung (C. icbikawai: Das Typus-Material ist nach Hiro'om Uchida [briefliche Mitteilung vom 6.1.1974] ausgetrocknet, und die Art wurde seitdem nicht wieder gefunden). Entscheidendes Gewicht wurde hierbei der Anwesenheit eines Scapulus beigemessen, der sich durch die Anhäufung von basitrichen Nesselkapseln im Ektoderm auszeichnet. Die Kombination dieser Merkmale ist meines Wissens niemals innerhalb der Sagartiidae beobachtet worden und rechtfertigt die Aufstellung einer neuen Gattung. UCHIDA (1960, p. 596) nennt allerdings als wichtigste Merkmale seiner Gattung Carcinactis das zahlreichere Vorkommen von Septen an der Basis und den relativ geringen Größenunterschied zwischen den inneren und den äußeren Tentakeln. Meiner Ansicht nach sollte diesen beiden Merkmalen nur eine untergeordnete Bedeutung bei der Abgrenzung der Gattung zugestanden werden, da ihre Variabilität nicht auszuschließen ist. Die Beschreibung von C. ichikawai weist Lücken auf, so daß es vorläufig nicht möglich ist, weitere Merkmale in die Gattungsdiagnose mit einzubeziehen. So fehlen Angaben über die Position der Cincliden und über den Typ jener Nesselkapseln, die im Scapulus gehäuft vorkommen. Auch das Cnidom kann einer taxonomischen Wertung noch nicht unterzogen werden, da sich bei der Untersuchung und Darstellung des Cnidoms von C. ichikawai vermutlich Fehler eingeschlichen haben (die Untersuchung der Nesselkapseln stammt nicht von UCHIDA; vgl. UCHIDA, 1960, p. 600).

Möglicherweise als Synonym von Carcinactis ist die monotypische Gattung Verrillactis ENGLANd (1971, p. 29) anzusehen. Diese Gattung wird von ENGLANd nur gegenïber älteren Sagartiiden-Gattungen, die im "Survey " von CARLGREN (1949) aufgeführt sind, abgegrenzt. Wenn man annimmt, daß ENGLAND (1971, p. 30) die Cincliden übersehen hat ("cinclids seem to be absent"), so bleiben nur noch zwei Merkmale der Typus-Art V. paguri (VerrmL, 1869), die bisher bei der Gattung Carcinactis nicht beobachtet wurden: (1) Ungeschlechtliche Vermehrung durch Lazeration. (2) Die inneren Tentakel können zu Fangtentakeln ungebildet werden. Beide Merkmale sind nicht als diagnostische Merkmale geeignet, da sie sporadisch bei vielen Arten der Acontiaria auftreten und darüber hinaus Abhängigkeit von ökologischen Gegebenheiten zeigen (vgl. RIEMANN-Zürneck, 1969, p. 188 und die in der vorliegenden Arbeit gemachten Be-merkungen zum Thema Lazeration, vgl. p. 93). Verrillactis paguri hat mit Carcinactis dolosa viele Merkmale gemeinsam, insbesondere jene, die an Calliactis erinnern. Beide Arten kommen gemeinsam mit Vertretern der Gattung Calliactis vor. Auch die von ENGLAND dargestellten Nesselkapseln haben in Typen und Abmessungen Ahnlichkeit mit jenen bei C. dolosa.

Es besteht die Möglichkeit, daß auch in anderen Sagartiiden-Gattungen sich Arten 
verbergen, die zu der Gattung Carcinactis zu rechnen sind. So zeigt zum Beispiel Actinotboe californica CARLGREN, 1940 [CARLGREN (1949) verzeichnet diese Art auch unter der Gattung Sagartiogeton] trotz lückenhafter Beschreibung mit Carcinactis dolosa Übereinstimmung in mehreren Merkmalen: Typen und Abmessungen der Nesselkapseln, Farbmuster und die Bevorzugung von Mollusken-Schalen als Siedlungs-Substrat.

Innerhalb der Sagartiiden steht Carcinactis der Gattung Anthotboe (CARLgren, 1938) am nächsten. Die Uberprüfung des Typus dieser Gattung, A. stimpsoni (VerriLl, 1868), am Zoologischen Museum in Kopenhagen (Fundort: Cape Barracouta 13. 12. 1929, leg. Mortensen, det. CARLgRen) ergab hinsichtlich der Anordnung der Gonaden und der Ausprägung der Muskulatur Gemeinsamkeiten.

\section{Gattung Phellia}

Pbellia exlex (McMurrich, 1904)

Carlgren (1928, p. 236), Carlgren (1949, p. 104); syn. Hormathia exlex McMurrich (1904, p. 279, Fig. 70, 71); syn. Synphellia exlex CarLGREN (1927, p. 71, Fig. 46, 47).

M a t e ria l. Ein Exemplar von der „WALTHER-HerwIG“-Expedition 1966, Station $450\left(36^{\circ} 05^{\prime} \mathrm{S} 53^{\circ} 45^{3} \mathrm{~W}, 100 \mathrm{~m}\right)$; Arbeitsnummer: $184 / 66$.

Ha bitus. Ovoid bis eiförmig, Tentakel kontrahiert, Fußscheibe schmaler als der Körperdurchmesser, leicht nach innen gewölbt. Mauerblatt in $z$ wei Regionen gegliedert: Scapus und Scapulus. Scapulus nackt, weiß bis blaßrosa gefärbt, in Form von 12 marginalen Wülsten die Körperöffnung des kontrahierten Tieres umgebend. Scapus dunkel gefärbt, mit einer fest mit dem Ektoderm verbundenen Kutikula. Tenaculi im obersten Abschnitt des Scapus am größten und dort in 12 Längsreihen angeordnet. Tenaculi mit flachen, chitinigen Plättchen bedeckt, die sich durch ihre kastanienbraune Färbung von der Umgebung abheben.

Die Höhe des Tieres beträgt $3,6 \mathrm{~cm}$, der Durchmesser $3,4 \mathrm{~cm}$, sein Gewicht ist $11,5 \mathrm{~g}$.

B a u p la n. Septen in 4 Cyklen vorhanden, zusätzlich einige winzige Septen des 5. Cyklus; Ausbildung und Größe des 4. Cyklus unregelmäßig. 1. und 2. Septencyklus vollständig, darunter je 2 Direktivenpaare. Die vier Direktivenpaare und Siphonoglyphen nicht einander gegenüberliegend; Muskulatur und Siphonoglyphen der beiden Direktivenpaare des 1. Cyklus größer als jene des 2. Cyklus (vgl. Abb. 5a). Im mittleren Abschnitt des Körpers sind die vollständigen Septen von relativ großen Septalstomata durchbrochen. Tentakel etwa 100, kurz und spitz mit schwacher Längsfurchung. Actinopharynx mit ca. 100 Längsfurchen und wenigen durch die Kontraktion entstandenen Querfalten. Das Tier besitzt keine Gonaden. Acontien außerordentlich dick und zahlreich; sie werden in einem Bündel aus der Körperöffnung ausgeworfen.

$\mathrm{H}$ is to log i e. (a) Cnidom (vgl. Abb. 5): Bei der Untersuchung der Nesselkapselausstattung eines einzelnen Tieres kann in einigen Geweben nicht ausgeschlossen werden, daß aus anderen Körperteilen verschleppte Nesselkapseln anwesend sind. Bei der tabellarischen Zusammenfassung der Nesselkapseln wird daher Scapulus und Scapus 
Tabelle 5

Abmessungen der Nesselkapseln von Phellia exlex

\begin{tabular}{|lll|}
\hline Körperregion & Nematocysten & Dimension $(\mu \mathrm{m})$ \\
\hline Mauerblatt & p-Mastigophoren & $14,5-22 \times 2,5-3$ \\
(Scapus + Scapulus) & Basitrichen 1* & $17-19,5 \times 3,5-4,5$ \\
& Basitrichen 2* & $8,5-11,5 \times 1,5$ \\
Tentakel & Spirocysten maximal & $39 \times 3$ \\
& p-Mastigophoren & $13-17 \times 2,5$ \\
& Basitrichen 1* & $22-27 \times 2-2,5$ \\
& Basitrichen 2* & $10-12 \times 1,5$ \\
Actinopharynx und unilobate & p-Mastigophoren 1* & $29-32,5 \times 3-4$ \\
Mesenterialfilamente & p-Mastigophoren 2* & $19,5-27 \times 3-4$ \\
& ?p-Mastigophoren 3* & $13-15,5 \times 4-4,5$ \\
& Basitrichen 1 & $27-30 \times 3$ \\
& Basitrichen 2* & $12-22 \times 1,5$ \\
Acontien & p-Mastigophoren* & $35-39 \times 3,5$ \\
& Basitrichen 1 & $41,5-47 \times 4-4,5$ \\
& Basitrichen 2* & $13-23,5 \times 1,5$ \\
\hline
\end{tabular}

sowie Pharynx und unilobate Mesenterialflamente nicht getrennt aufgeführt. Mit Sicherheit konnte jedoch festgestellt werden, daß die kleine p-Mastigophore des Mauerblattes nur im Scapulus und die birnenförmige, als „?-p-Mastigophore“ (vgl. Abb. 5) nur in den Mesenterialfilamenten vorkommt. Die mit * bezeichneten Nesselkapseln wurden bisher bei dieser Art nicht gefunden (vgl. CARLGREN, 1927, p. 31).

(b) Muskulatur: Retraktoren des 1. Septencyklus kräftig entwickelt, mit hohen Mesogloeafortsätzen, diffus-circumskript, die des 2. Cyklus schwächer, diffus; an den Cyklen 3 und 4 keine Retraktoren. Sphinkter mesogloeal, relativ lang (ca. $2 \mathrm{~cm}$ ). Parietalmuskulatur vorhanden. (Da von dem einzigen Exemplar keine Schnitte angefertigt wurden, kann keine komplette Beschreibung der Muskulatur gegeben werden.)

Verbre it ung. Die beiden Typus-Exemplare, die bisher das einzige Material dieser Art darstellten, stammen aus dem Litoral der chilenischen Küste (ca. $42^{\circ} \mathrm{S} 73^{\circ} \mathrm{W}$ ), während der Fundort von "WALther Her wIG" im Bereich des „Rio-de-la-Plata“-Deltas in $100 \mathrm{~m}$ Tiefe liegt.

\section{FAMILIE METRIDIIDAE}

\section{Gattung Metridium}

Die Typus-Art der Gattung Metridium senile L. zeichnet sich aus durch eine bemerkenswerte Variabilität von Merkmalen, die sonst innerhalb der Acontiaria einen hohen taxonomischen Rang besitzen und zum Beispiel zur gegenseitigen Abgrenzung der Familien Hormathiidae und Sagartiidae benutzt werden. Als ein wesentliches diagnostisches Merkmal der Familie wertet HaND (1955, p. 190) das Vorhandensein von b-Mastigophoren in den Acontien. Die elektronenoptischen Untersuchungen von WESTFALL (1965, p. 79) machen es wahrscheinlich, daß es sich bei der als „b-Mastigophore“ 
bezeichneten Nesselkapsel um eine Größenvariante der Basitrichen handelt; damit verliert auch dieses Merkmal seine bisherige taxonomische Bedeutung.

Als wichtigstes Merkmal für die Zuordnung von Arten zu der Gattung Metridium sehe ich die Gliederung des Mauerblattes in zwei Regionen, Scapus und Kapitulum. Wichtig erscheint mir hierbei, daß das Kapitulum im Sinne von CARLGREN \& STEPHENSON $(1928, p .16)$ definiert wird als dünnwandige Region, die keinen Sphinkter enthält, Modifizierungen des Ektoderm und der entodermalen Ringmuskulatur zeigt und sich oft auch farblich vom Scapus abhebt.

Metridium senile subsp. lobatum (CARLGREN, 1899) nov. comb. nov. grad.

Synonyme: Sagartia lobata CARlgren (1899, pp. 36-37, Fig. 2); Metridium parvulum McMurRIcH (1904, pp. 276--278, Fig. 66-68).

$\mathrm{M}$ a te rial. Metridium senile subsp. Lobatum wurde an sechs Stationen der „WALther-Herwig “-Expeditionen gesammelt (Tab. 6); das Material umfaßt neun adulte Tiere und ca. 30 durch Lazeration entstandene Jungtiere.

Vergleichende Untersuchungen wurden an Metridium senile aus dem Nordatlantik durchgeführt. Hierfür stand ein kleines Tier (Gewicht 1,8 g, keine Gonaden) aus dem Zoologischen Museum Kopenhagen (Fundort: Frederikshavn) und ein besonders großes Exemplar (Gewicht 250 g) aus dem Zoologischen Museum Hamburg (Nr. C 7152, Fundort: Südwest-Island) zur Verfügung.

Tabelle 6

Material von Metridium senile subsp. lobatum

\begin{tabular}{|c|c|c|c|c|c|c|c|}
\hline $\begin{array}{l}\text { Tier } \\
\text { Nr. }\end{array}$ & $\begin{array}{l}\text { WALTHER- } \\
\text { HER WIG- } \\
\text { Expedition }\end{array}$ & $\begin{array}{l}\text { Station } \\
\text { Nr. }\end{array}$ & Koordinaten & $\begin{array}{l}\text { Tiefe } \\
(\mathrm{m})\end{array}$ & $\begin{array}{l}\text { Besiedeltes } \\
\text { Substrat }\end{array}$ & $\begin{array}{c}\text { Anzahl } \\
\text { der Tiere }\end{array}$ & $\begin{array}{l}\text { Abb. } \\
\text { Nr. }\end{array}$ \\
\hline $188 / 66$ & 1966 & 374 & $44^{\circ} 20^{\prime} \mathrm{S} 62^{\circ} 52^{\prime} \mathrm{W}$ & 95 & Stein & 1 & \\
\hline 182 & $1970 / 71$ & 182 & $45^{\circ} 32^{\prime} \mathrm{S} 63^{\circ} 59^{\prime} \mathrm{W}$ & 107 & $\begin{array}{l}\text { Schale einer } \\
\text { lebenden Pecten }\end{array}$ & $2+$ juv. & $5 b$ \\
\hline 208 & $1970 / 71$ & 208 & $49^{\circ} 32^{\prime} \mathrm{S} 66^{\circ} 30^{\prime} \mathrm{W}$ & 98 & - & 1 & $5 \mathrm{~d}, \mathrm{e}, \mathrm{f}$ \\
\hline 314 & $1970 / 71$ & 314 & $45^{\circ} 03^{\prime} \mathrm{S} 63^{\circ} 00^{\prime} \mathrm{W}$ & 102 & $\begin{array}{l}\text { Brachiopoden- } \\
\text { Schale }\end{array}$ & $1+$ juv. & $5 c, g$ \\
\hline 304 & $1970 / 71$ & 304 & $47^{\circ} 00^{\prime} \mathrm{S} 65^{\circ} 00^{\prime} \mathrm{W}$ & 85 & - & 2 & \\
\hline 305 & $1970 / 71$ & 305 & $47^{\circ} 00^{\prime} \mathrm{S} 64^{\circ} 00^{\prime} \mathrm{W}$ & 120 & Pecten & $2+$ juv. & \\
\hline
\end{tabular}

H a bi t u s. (a) Körperform und Größe (Abb. 5b-d): Mittelgroße Unterart, deren Fußscheibe stets ausgebreitet und am Limbus auffällig gelappt ist; die Tiere Nr. 188/66, 182, 305 und 314 zeigen ungeschlechtliche Vermehrung durch Lazeration (Abb. 5b). Mit Ausnahme des Tieres Nr. 314, das mit ausgestrecktem Tentakelkranz fixiert wurde, sind die Tiere stark kontrahiert und haben die bei $M$. senile bekannte flache Kegelform, bei M. senile subsp. lobatum bleibt in allen Fällen ein Teil der Ten- 

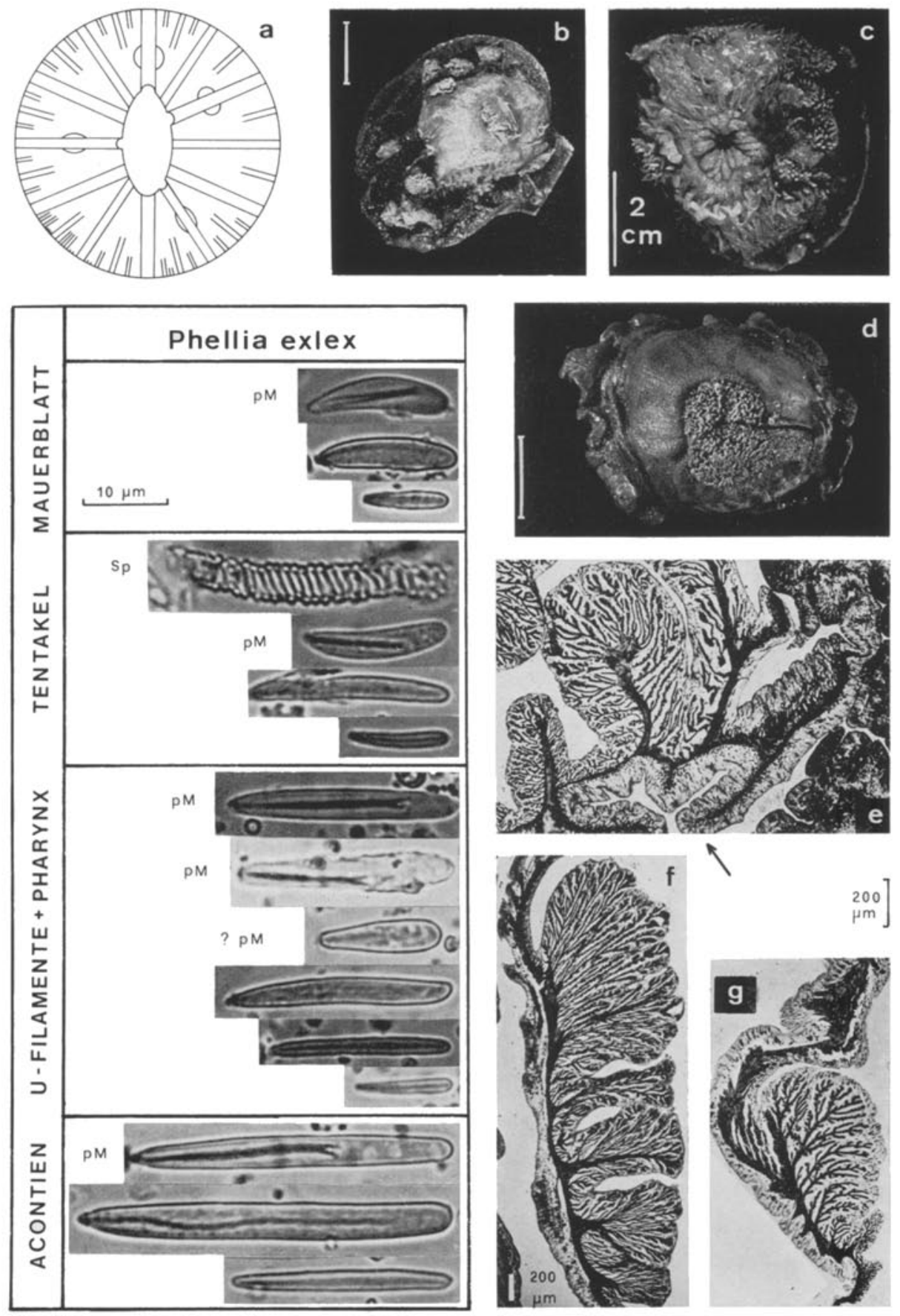

Abb. 5: a Pbellia exlex. Nesselkapselausstattung des einzigen Tieres (184/66) und Bauplan. $b$ Metridium senile subsp. lobatum. Tier Nr. $182 \mathrm{mit}$ Jungtieren, die durch Lazeration entstanden; $c$ Tier Nr. 314; $d$ Tier Nr. 208; $e$ Retraktor des 3. Septencyklus (Nr. 208) mit Mesogloeaneubildung auf der retraktorabgewandten Seite (Pfeil); f Retraktor des 1. Septencyklus (Tier Nr. 208); g Retraktor des 1. Septencyklus eines kleineren Tieres (Nr. 314) 
takel an der Körperöffnung sichtbar. Tentakelkranz im ausgestreckten Zustand (Tier Nr. 314) im Durchmesser größer als der des Körpers, Tentakel auf mehreren Loben der Mundscheibe (Abb. 5c).

(b) Mauerblatt: Gegliedert in Scapus und Kapitulum; zwischen den beiden Körperregionen liegt ein Ringwulst (collar). Mauerblatt glatt, dünnwandig, bei großen und stark kontrahierten Tieren (Nr. 208) mit feinen Runzeln (Abb. 5d). Cincliden am Limbus oder in Limbusnähe, ihr Vorhandensein ist nur am Austreten von Acontien erkennbar.

(c) Gewicht des größten Tieres: $36 \mathrm{~g}$ (Nr. 208).

$\mathrm{B}$ a u $\mathrm{p}$ l a n. Scapus bis $1 \mathrm{~mm}$ dick, Kapitulum nur wenig dünner. Pharynx zartwandig, mit wenigen Längswülsten, die vermutlich in gleicher Zahl wie die Lippenwülste vorhanden sind. Ein bis zwei breite Siphonoglyphen. Tentakel in mindestens 7 Cyklen (ca. 400 Stïck), die ersten 3 Cyklen (bei Tier Nr. 314) regelmäßig hexamer auf der Mundscheibe angeordnet, sehr dünnwandig und relativ groß (bis $1,5 \mathrm{~cm}$ lang). Die übrigen Tentakel sind klein und spitz und am Rande der Mundscheibe angeordnet (Abb. 5c). Fangtentakel bei keinem der Tiere gefunden.

Septen in 6 Cyklen vorhanden, die ersten drei Cyklen regelmäßig hexamer, die restlichen unregelmäßig angeordnet. Erster oder erster und zweiter Cyklus vollständig, meist steril (bei 'Tier 188/66 einige vollständige Septen fertil). Gonaden sonst an den Cyklen 3 bis 5 gemeinsam mit trilobaten Filamenten, unterhalb der Gonadenregion unilobate Filamente. Acontien an den Cyklen 1 bis 5. Sechster Septencyklus ohne Anhangsorgane. Ein bis zwei Direktivenpaare vorhanden. Septen in der Regel sehr zart (bei Tier Nr. 208 etwas dicker), die vollständigen sind mit Oral- und Marginalstomata versehen.

$\mathrm{H}$ is t o log i e. (a) Cnidom (vgl. Tab. 7): Die Nesselkapselausstattung wurde bei allen adulten Tieren mit Ausnahme des Tieres Nr. 188/66 untersucht. $M$. senile subsp. lobatum unterscheidet sich von $M$. senile durch das zusätzliche Vorkommen von kleinen p-Mastigophoren in den Tentakeln; diese Nesselkapsel befindet sich im Entoderm und ist auch auf Schnittpräparaten zu erkennen.

(b) Muskulatur: Längsmuskulatur der Tentakel ektodermal, Radialmuskulatur der Mundscheibe ektodermal, kräftig entwickelt. Retraktoren nur an den Septencyklen 1 bis 3 vorhanden, bei Tier Nr. 208 auch am 4. Cyklus. Retraktoren auf den Septen zentral liegend. Bei juvenilen Tieren (Körperdurchmesser $8 \mathrm{~mm}$ ) Retraktoren circumskript diffus, bei größeren Tieren nimmt die Ausdehnung des Retraktors auf dem Septum und die Höhe der Mesogloeafortsätze mit der Größe der Septen zu (Abb. 5f). Darüber hinaus wächst bei größeren Tieren die Tendenz, einen circumskripten Retraktor auszubilden, der in dieser Form unabhängig vom Septencyklus auftritt (bei Tier Nr. 208 auch am 4. Septencyklus). Bemerkenswert ist in diesem Zusammenhang, daß bei dem größten - und in vielen Merkmalen etwas aberranten - Tier (Nr. 208) auf der retraktorabgewandten Seite des Septums Mesogloeafortsätze vorhanden sind, die anscheinend dort von kleinen, massenhaft vorhandenen Zellen gebildet werden (Abb. 5e). Parietalmuskulatur nicht vorhanden. Ringmuskulatur des Scapus schwach, Mesogloeafortsätze auf Längsschnitten 20 bis $30 \mu \mathrm{m}$ hoch, im Bereich des Sphinkters abgeschwächt und im Kapitulum nicht vorhanden. Sphinkter mesogloeal, im obersten Abschnitt des Scapus in der dort etwas verdickten Mesogloea. Struktur des Sphinkters fein 


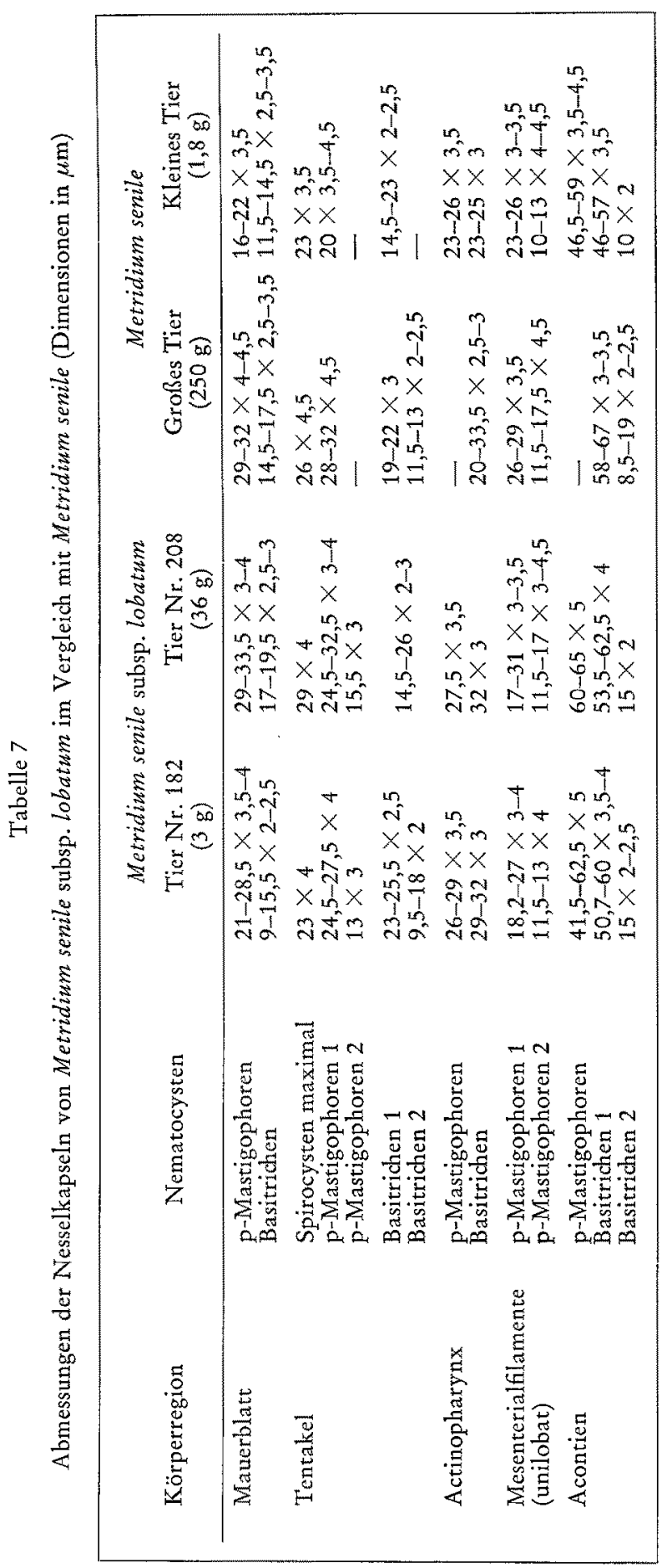


alveolär, bei Tier Nr. 208 verschmelzen mehrere kleine Alveolen zu einer größeren. Alveolenfreie Mesogloeaschicht nur zum Ektoderm hin vorhanden. Länge des Sphinkters bei mittelgroßen Tieren (Nr. 314, 182, 304) 1,5 bis $2 \mathrm{~mm}$ lang, bei Tier Nr. 208 4,5 $\mathrm{mm}$ und bei einem juvenilen Tier (Körperdurchmesser $8 \mathrm{~mm}$ ) 0,8 $\mathrm{mm}$. Die Länge des Sphinkters bei den zwei Vergleichstieren von $M$. senile ist bei dem kleinen Tier $1,5 \mathrm{~mm}$ und bei dem großen Individuum $8,1 \mathrm{~mm}$.

(c) Mesogloea des Scapus auf Querschnitten mit parallel angeordneten Fasern, hier wie auch in der Mesogloea des Pharynx besonders zahlreiche acidophile Einschlüsse.

(d) Gonaden bei allen Tieren vorhanden, mit Ausnahme des größten Tieres (Nr. 208) handelt es sich um Ovarien. Die Eizellen haben auf Schnitten einen Durchmesser von ca. $100 \mu \mathrm{m}$. Da gutentwickelte Gonaden sowohl im Suidwinter wie auch im Südsommer vorhanden sind, läßt sich über den Zeitpunkt der geschlechtlichen Fortpflanzung nichts aussagen.

V e r b r e it u n g. Gegenwärtig unterscheidet man bei Metridium senile die nordatlantische Nominatform und die im Nordpazifik vorkommende Metridium senile subsp. fimbriatum (Verrill, 1865). Die vorliegende Beschreibung ist der erste Nachweis einer Unterart von Metridium senile auf der südlichen Hemisphäre. Die neun Exemplare wurden vor der Küste Südargentiniens zwischen dem $44^{\circ}$ und dem $50^{\circ}$ südlicher Breite gesammelt, wobei alle Fundorte nahe der 100-m-Tiefenlinie liegen. Das Vorkommen in größeren Tiefen kann mit großer Wahrscheinlichkeit ausgeschlossen werden, da die Unterart an keiner der zahlreichen tieferen "WALTHER-HERWIG"-Stationen gefunden wurde. Es ist unbekannt, ob Metridium senile subsp. lobatum vor der Küste Argentiniens auch in das Flachwasser oder sogar ins Litoral vordringt; an der chilenischen Küste kommt $M$. senile subsp. lobatum im Flachwasser zwischen 8 und $35 \mathrm{~m}$ vor (CARLGRen, 1899: „Sagartia lobata“; MCMURrICH, 1904: „Metridium parvulum"). Die wenigen bekannten Fundortdaten lassen die Vermutung zu, daß diese Unterart auf der Südhalbkugel ähnliche ökologische Ansprïche stellt wie $M$. senile in ihrem bisher bekannten circumpolaren Verbreitungsgebiet auf der nördlichen Hemisphäre. Wie bei $M$. senile und $M$. senile subsp. fimbriatum handelt es sich auch hier um eine Flachwasser-Aktinie, die in subpolaren, kalten bis temperierten Meeresteilen lebt. Metridium senile subsp. lobatum wurde gemeinsam mit den Aktinien Antholoba achates (DANA 1849), Choriactis laevis (CARLgReN 1899) und Actinostola sp. gefunden.

Vergleichende Untersuchungen und Diskussion. Die juvenilen Exemplare der von "WALTHER HER WIG" gesammelten Tiere zeigen Übereinstimmung mit der Beschreibung von "Metridium parvulum" (McMurrich, 1904). McMurRICH stellte bereits fest (p. 278), daß M. parvulum in vielerlei Hinsicht Sagartia lobata (CARLGREN, 1899) ähnelt. Die unterschiedliche Anzahl der vollständigen Septen bewog MCMurRich jedoch, beide Arten getrennt bestehen zu lassen. Nach Untersuchungsergebnissen an $M$. senile (vgl. StepHenson, 1935, pp. 223-224) ist die Anzahl der vollständigen Septen variabel und kann nur noch unter Vorbehalt als gattungsdiagnostisches Merkmal benutzt werden. Einer Synonymisierung der beiden Arten steht damit nichts mehr im Wege.

Die größeren "WALTHER-HER WIG "-Exemplare und insbesondere das Tier Nr. 208 zeigen gegenüber den Beschreibungen Carlgrens und McMurrichs Abweichungen, 
die es notwendig machten, vergleichende Untersuchungen an nordatlantischem Material von $M$. senile durchzuführen. Ergänzt wurde dieser Vergleich durch die Einbeziehung von früheren Beschreibungen der nördlichen Unterarten von Metridium senile, wobei insbesondere Untersuchungen zur Variabilität von Merkmalen zu nennen sind (STEPHENSON, 1935, pp. 214-232; HAND, 1955, pp. 191-210; Averincev, 1967, pp. 72-75).

Das Ergebnis dieser Studien ist, daß die yor den Küsten Südamerikas bisher gefundenen Exemplare der Gattung Metridium weitgehend identisch sind mit Metridium senile der nördlichen Hemisphäre. Ubereinstimmung wurde gefunden in der Nesselkapselausstattung und der Variabilität der Nesselkapseln, in Habitus und Bauplan, in Lage und Struktur der Muskulatur und in den ökologischen Daten der Fundorte. Die südamerikanischen Tiere sollten trotz dieser Übereinstimmung als Unterart geführt werden, da sie einige morphologische Besonderheiten besitzen. Dies ist die gegenüber der Nominatform relativ geringe Körpergröße, der ungewöhnlich stark gelappte Rand der Fußscheibe, die bei allen auf festem Untergrund sitzenden Tieren zu beobachtende Lazeration der Fußscheibe, die kleinen p-Mastigophoren im Entoderm der Tentakel und die größere Neigung der Retraktor-Muskulatur zur circumskripten Form. Es ist jedoch auch möglich, daß diese Abweichungen durch Unterschiede in den ökologischen Gegebenheiten hervorgerufen werden. So stellte zum Beispiel RawLinson (1934, p. 904) bei der nordatlantischen $M$. senile die Neigung zu $Z_{\text {wergwuchs }}$ und zu Lazeration im Zusammenhang mit der Verschlechterung von Umweltbedingungen fest.

Carlgren nennt $(1949$, p. 106) neben der Typus-Art Metridium senile noch zweivon ihm als fraglich angesehene - Arten: $M$. parvulum McMurrick, 1904 und $M$. canum Stuckey, 1914. Eine weitere Art (M. exile, lapsus: exilis) wurde von HaND (1955) hinzugefügt. Nachdem $M$. canum als Mimetridium canum zu der Familie Acontiophoridae gestellt wurde (HAND, 1961, p. 82) und $M$. parvulum in der vorliegenden Arbeit als ein Synonym von $M$, senile angesehen wird, bleibt in der Gattung Metridium nur noch $M$. exile neben der Typus-Art zurück*. Diese Art muß jedoch - nach den an $M$. senile und $M$. senile subsp. lobatum gewonnenen Erfahrungen - als Synonym von Metridium senile subsp. fimbriatum angesehen werden. Die in der Differentialdiagnose (HAND 1955, p. 210) genannten Charakteristika liegen entweder innerhalb der Variationsbreite von $M$. senile (Länge des Sphinkters) oder sie sind auf die geringe Größe der Tiere zurückzuführen (Form der Retraktoren). Die besondere Größe der Kapitulum-Nesselkapseln kann nach Averincev (1967, pp. 73-74) auch bei M. senile subsp. fimbriatum vorkommen. Auch die anderen genannten Unterschiede des Cnidoms können nicht zur Differenzierung benutzt werden, da die Unterscheidung von b-Mastigophoren und Basitrichen einerseits sowie Amastigophoren und p-Mastigophoren andererseits für taxonomische Zwecke nicht mehr gerechtfertigt erscheint (vgl. RIEMANNZüRNECK, 1973, p. 278). Eine Abgrenzung von M. exile ist demnach nicht mehr möglich, und es muß angenommen werden, daß es sich hier um eine zwerghafte Küstenform von $M$. senile subsp. fimbriatum handelt, wie man sie auch von $M$. senile aus dem Nordatlantik kennt (STEPHENSON, 1935, p. 222).

Nachdem nunmehr der Nadwweis geführt werden konnte, daß $M$. senile auch auf

* Auf die von Averuncev (1967, pp. 75-76) beschriebene Metridium sp. sei hier aufmerksam gemacht. 
der Südhalbkugel vorkommt, scheint es verwunderlich, daß dies nicht schon von früheren Sammlern und Autoren festgestellt oder vermutet wurde. Für eine physiologisch so anpassungsfähige und widerstandsfähige Art wie Metridium senile sollte es besonders leicht sein, angeheftet an Schiffen die Warmwasserregion der Erde zu überqueren, zumal diese Aktinie eine Vorliebe zur Besiedlung schwimmender Gegenstände zeigt.

\section{ZUSAMMENFASSUNG}

1. Im vorliegenden zweiten Teil der Bearbeitung des im Schelfgebiet vor Argentinien gesammelten Aktinien-Materials werden drei Arten der Familie Sagartiidae und eine Unterart der Familie Metridiidae beschrieben.

2. Die Untersuchung von Choriactis laevis (CARLGREN, 1899) führt neben neuen anatomisch-histologischen Ergebnissen zu neuen Erkenntnissen der Fortpflanzungsbiologie und Larvalentwicklung, die von allgemeinem Interesse sind: (a) Die Sexualverhältnisse bei $C$. laevis sind ungewöhnlich und waren bei Aktinien bisher unbekannt: Die Art ist zwittrig mit komplementären Männchen. (b) C. laevis ist eine brutpflegende Sagartiide; die Entwicklungsstadien befinden sich bis zur Planula im Gastralraum des Muttertieres. (c) Die Planula, deren Bauplan dem „Edwardsia-Stadium“ entspricht, besitzt einen bisher bei Aktinienlarven nicht beobachteten Habitus: Schon vor Anlage der Tentakel hat sich am aboralen Pol ein Haftorgan gebildet, das massenhaft Spirocysten enthält und an dessen Aufbau nur das Ektoderm beteiligt ist.

3. Carcinactis dolosa n. sp. ist eine Sagartiide, die große Ahnlichkeit mit Vertretern der Gattung Calliactis (Fam. Hormathiidae) besitzt. Die Beschreibung schließt eine Diskussion der Gattung Carcinactis UCHIDA, 1960 und der Gattung Verrillacis ENGLAND, 1971 ein.

4. Für Pbellia exlex (McMurrich, 1904) werden Beschreibungen von Bauplan und Cnidom gegeben.

5. Die Beschreibung von Metridium senile subsp. lobatum (CARLGREN, 1899) nov. comb. nov. grad., syn. Sagartia lobata CARLGReN, 1899, belegt den ersten Nachweis einer Unterart von Metridium senile auf der südlichen Hemisphäre. Vergleichend untersucht wurden zwei Tiere der nordatlantischen Metridium senile. In der Diskussion wird der Status der Gattung Metridium behandelt; Metridium exile Hand, 1955 wird als ein Synonym ron Metridium senile subsp. fimbriatum (VERRILL) angesehen.

\section{ZITIERTE LITERATUR}

AverinceV, V. G., 1967. Actiniaria of the Possjet Bay of the Sea of Japan. Issled. Fauny Morei 5, 62-77.

CARLgReN, O., 1893. Studien über nordische Aktinien. K. svenska VetenskAkad. Handl. 25, $1-148$.

- 1899. Zoantharien. Hamburger Magelhaensische Sammelreise 4, 1-47.

- 1925. On the actiniarian family Amphianthidae. Ark. Zool. 17, 1-6.

- 1927. Actiniaria and Zoantharia. Further zool. Results Swed. Antarct. Exped. 2, 1-102.

- 1928. Actiniaria der Deutschen Tiefsee-Expedition. Wiss. Ergebn. dt. Tiefsee-Exped. „Valdivia" 22, 126-266. 
- 1932. Die Ceriantharien, Zoantharien und Actiniarien des arktischen Gebietes. Fauna arct. $6,254-266$.

- 1940. Actiniaria from the Gulf of California. Zoologica, N. Y. 25, 210-219.

- 1949. A survey of the Ptychodactiaria, Corallimorpharia and Actiniaria. K. svenska VetenskAkad. Handl. (Ser. 4) 1, 1-121.

- 1951. The actinian fauna of the Gulf of California. Proc. U. S. natn. Mus. 101, 415-449.

- \& Stephenson, T. A., 1928. The British Edwardsidae. J. mar. biol. Ass. U. K. 15, 1-31.

DanA, J. D., 1848-1849. Zoophytes. United States exploring expedition. Philadelphia, 1-740.

England, K. W., 1971. Actiniaria from Muroroa atoll, Tuamotu, Polynesia. Cah. Pacif. 15, $23-39$.

Haddon, A. C., 1889. A revision of the British Actiniae I. Scient. Trans. R. Dubl. Soc. 4, 297-361.

Hand, C., 1955. The sea anemones of Central California. Part III. The acontiarian anemones. Wasmann J. Biol. 13, 189-215.

- 1961. Two new acontiate New Zealand sea anemones. Trans. R. Soc. N. Z. (Zool.) 1, 75-89.

McMurrich, J. P., 1904. The Actiniae of the Plate Collection. Zool. Jb. (Suppl.) 6, 215-306.

PAx, F., 1922. Diagnosen neuer Actiniarien aus der Ausbeute der Deutschen und der Französischen Südpolar-Expedition. Zool. Anz. 54, 74-92.

- 1923. Antarktische Aktinien aus der Ausbeute der „Pourquoi pas“. Zool. Jb. (System. Okol. Geogr. Tiere) 47, 1-28.

Ratrinson, R., 1934. A comparative study of Metridium senile var. dianthus and a dwarf variety of this species occuring in the river Mersey. J. mar. biol. Ass. U. K. 19, 901-919.

Riemann-Zürneck, K., 1969. Sagartia troglodytes. Biologie und Morphologie einer schlickbewohnenden Aktinie. Veröff. Inst. Meeresforsch. Bremerh. 12, 169-230.

- 1973. Actiniaria des Südwestatlantik. I. Hormathiidae. Helgoländer wiss. Meeresunters. 25, 273-325.

Srmon, J. A., 1892. Ein Beitrag zur Anatomie und Systematik der Hexactinien. Diss. München, $106 \mathrm{pp}$.

Stephenson, T. A., 1920. On the classification of Actiniaria. Part I. Forms with acontia and forms with a mesogloeal sphinkter. Q. Jl microsc. Sci 64, 425-574.

- 1928. The British sea anemones. Ray Soc., London 1, 1-148.

- 1935. The British sea anemones. Ray Soc., London 2, 1-426.

Stuckex, F. G. A., 1914. Description of a collection of actinians from Kermadec Islands. Trans. N. Z. Inst. 46, 132-134.

UchIDA, T., 1960. Carcinactis ichikawai, an actiniarian commensal with the crab Dorippe granulata. Jap. J. Zool. 12, 595-601.

Westrall, J. A., 1965. Nematocysts of the sea anemone Metridium. Am. Zool. 5, 377-393.

Anschrift der Autorin: Dr. Karin RIEMAnN-Zürneck

Institut für Meeresforschung

D-285 Bremerhaven

Am Handelshafen 12

Bundesrepublik Deutschland 